\title{
On a Higher Order Accurate Fully Discrete Galerkin Approximation to the Navier-Stokes Equations*
}

\author{
By Garth A. Baker, Vassilios A. Dougalis and Ohannes A. Karakashian
}

\begin{abstract}
We consider approximating the solution of the initial and boundary value problem for the Navier-Stokes equations in bounded two- and three-dimensional domains using a nonstandard Galerkin (finite element) method for the space discretization and the third order accurate, three-step backward differentiation method (coupled with extrapolation for the nonlinear terms) for the time stepping. The resulting scheme requires the solution of one linear system per time step plus the solution of five linear systems for the computation of the required initial conditions; all these linear systems have the same matrix. The resulting approximations of the velocity are shown to have optimal rate of convergence in $L^{2}$ under suitable restrictions on the discretization parameters of the problem and the size of the solution in an appropriate function space.
\end{abstract}

1. Introduction. Let $\Omega$ be a bounded domain in $R^{N}, N=2$ or 3 , with a smooth boundary $\partial \Omega$. Given $0<T<\infty$, we seek an $R^{N}$-valued function $u=\left(u_{1}, \ldots, u_{N}\right)$ (the velocity), and a real-valued function $p$ (the pressure), defined for $(x, t) \in \bar{\Omega} \times$ $[0, T]$ and satisfying the Navier-Stokes equations

$$
\left\{\begin{array}{l}
u_{t}-\nu \Delta u+(u \cdot \operatorname{grad}) u+\operatorname{grad} p=f \text { in } \Omega \times(0, T] \\
u=0 \text { on } \partial \Omega \times[0, T] \\
\operatorname{div} u=0 \text { in } \Omega \times[0, T] \\
u(x, 0)=u^{0}(x) \text { in } \bar{\Omega},
\end{array}\right.
$$

where $f$ is a given $R^{N}$-valued function defined on $\Omega \times[0, T], u^{0}$ is a given $R^{N}$-valued function defined on $\bar{\Omega}$ with $u^{0}=0$ on $\partial \Omega$ and $\operatorname{div} u^{0}=0$ in $\Omega$ and $\nu>0$ is a constant (the coefficient of the kinematic viscosity). It will be assumed that the data of the problem (1.1) are such that (1.1) has a unique solution pair $[u, p]$ (with $p$ being unique up to an additive constant) sufficiently smooth so that the convergence results hold. We refer the reader to the books by Ladyzhenskaya [12], Lions [15], and Temam [18] for existence, uniqueness, and regularity results for (1.1).

Our goal in this work is to construct and analyze a fully discrete Galerkin (finite element) method for the approximation of the solution of (1.1). Our method is based on a nonstandard Galerkin approximation in the space variables which uses finitedimensional subspaces, the elements of which are not required to be divergence-free.

Received August 19, 1981; revised October 29, 1981 and February 18, 1982.

1980 Mathematics Subject Classification. Primary 65N30; Secondary 65M15, 35Q10.

* Work supported in part by USARO Grant No. DAAG29-80-K-0056 and in part by a University of Tennessee Faculty Research Award. The first author acknowledges the support of the Institute for Higher Education, Saint George's, Grenada, West Indies. 
For the time discretization we shall use the third order accurate, three-step backward differentiation method coupled with a suitable extrapolation procedure for the nonlinear terms so that only one linear system (with the same matrix) will have to be solved at each time step. The use of a three-step method necessitates providing three initial conditions (starting values) for the scheme. We generate these initial conditions by appropriate single-step methods and extrapolation procedures; their construction involves the solution of five linear systems with the same matrix as the one associated with the linear systems of the subsequent time-stepping. Hence only one matrix need be formed (and, e.g., $L U$-decomposed once if the systems are solved by a direct method) in the whole computation.

To this end we begin by introducing notation and the appropriate function spaces that we shall use. For integer $s \geqslant 0$ and real $1 \leqslant p \leqslant \infty$ we denote by $W_{p}^{s}=W_{p}^{s}(\Omega)$ the (real) Sobolev spaces, defined in the customary way, of scalar, real-valued functions defined on $\Omega$ and let $|\cdot|_{s, p}$ denote the associated norms. We put $H^{s}=W_{2}^{s}$ and let $|\cdot|_{s},\langle\cdot, \cdot\rangle_{s}$ denote the associated norm, resp. inner product. For $s=0$ we denote the norm on $W_{p}^{0}=L^{p}$ by $|\cdot|_{L^{p}}$ and, in particular, on $L^{2}$ by $|\cdot|$ and the associated $L^{2}$-inner product by $\langle\cdot, \cdot\rangle$. As usual, we let $\stackrel{\circ}{H}^{1}$ be the space of those functions in $H^{1}$ which vanish on $\partial \Omega$ in the sense of trace. We let $\mathbf{H}^{s}=\left(H^{s}\right)^{N}$ be the space of $R^{N}$-valued functions $u=\left(u_{1}, \ldots, u_{N}\right)$ defined on $\Omega$ such that $u_{i} \in H^{s}$, $1 \leqslant i \leqslant N$. Correspondingly, we put $\stackrel{\circ}{H}^{1}=\left(\stackrel{H}{H}^{1}\right)^{N}$ and equip $\mathbf{H}^{s}$ with the inner product $(u, v)_{s}=\sum_{i=1}^{N}\left\langle u_{i}, v_{i}\right\rangle_{s}$, generating the product norm $\|\cdot\|_{s}=(\cdot, \cdot)_{s}^{1 / 2}$. On $\mathbf{L}^{2}=\left(L^{2}\right)^{N}$, the inner product, resp. norm, is denoted by $(\cdot, \cdot)$, resp. $\|\cdot\|$. We shall also use the Banach spaces $\mathbf{L}^{\infty}=\left(L^{\infty}\right)^{N}, \mathbf{W}^{1, \infty}=\left(W^{1, \infty}\right)^{N}$, which we equip with the norms $\|v\|_{\infty}=\max _{1 \leqslant i \leqslant N}\left|v_{i}\right|_{L^{\infty}},\|v\|_{1, \infty}=\max _{1 \leqslant i \leqslant N}\left|v_{i}\right|_{1, \infty}$, respectively, and the quotient space $H^{s} / R^{1}$, equipped with the norm $|v|_{H^{s} / R^{1}}=\inf _{c \in R^{1}}|v+c|_{s}$. Finally, if $v:[0, t] \rightarrow X$ is a (strongly) measurable map with values in a Banach space $\left\{X,\|\cdot\|_{X}\right\}$, we let $\|v\|_{L^{p}(0, t ; X)}=\left(\int_{0}^{t}\|v(\tau)\|_{X}^{p} d \tau\right)^{1 / p}$ for $1 \leqslant p<\infty$, and $\|v\|_{L^{\infty}(0, t ; X)}=\operatorname{ess} \sup _{0 \leqslant \tau \leqslant t}\|v(\tau)\|_{X}$. In case $t=T$ we shall write $L^{p}(0, t ; X)$ as $L^{p}(X), 1 \leqslant p \leqslant \infty$.

For $u, v \in \mathbf{H}^{1}$ we define the bilinear form

$$
a(u, v)=\sum_{i, j=1}^{N} \int_{\Omega} \frac{\partial u_{i}}{\partial x_{j}} \frac{\partial v_{i}}{\partial x_{j}} d x
$$

for which it is well known that there exists a constant $C_{a}=C_{a}(\Omega)$ such that

$$
\begin{cases}a(u, v) \leqslant\|u\|_{1}\|v\|_{1} & \forall u, v \in \mathbf{H}^{1}, \\ C_{a}\|u\|_{1}^{2} \leqslant a(u, u), & \forall u \in \stackrel{\mathbf{H}}{ }^{1} .\end{cases}
$$

We now consider approximating the solution $[u, p]$ of $(1.1)$ by a Galerkin-type method. For the space discretization we shall adopt the Lagrange multiplier technique of [2] that allows the use of subspaces, the elements of which are not necessarily divergence-free. A similar technique was originally proposed by Babuška [1] for the stationary (linear) Stokes equations and was analyzed by Falk in [8]; cf. also the works of Crouzeix and Raviart [5], and of Jamet and Raviart [10].

For integer $r \geqslant 2$ and for $0<h<1$ consider a family $\mathbf{S}_{h}^{r}=\mathbf{S}_{h}^{r}(\Omega)$ of finite-dimensional subspaces of $\stackrel{\mathbf{H}}{ }^{1}$ in which approximations to the velocity will be sought. (In [2] these subspaces are not required to satisfy the essential boundary conditions-they 
are just subspaces of $\mathbf{H}^{1}$. To simplify matters we require here that $\mathbf{S}_{h}^{r} \subset \stackrel{\circ}{\mathbf{H}}^{1}$. See Remark 4.4 for the nonconforming case.) $\mathbf{S}_{h}^{r}$ will consist of ordered $N$-tuples of piecewise polynomials of degree at most $r-1$ defined on a quasiuniform partition of $\bar{\Omega}$ and satisfying, for some constant $C$ independent of $h$, the approximation property

$$
\inf _{\chi \in \mathbf{S}_{h}^{r}}\left(\|u-\chi\|+h\|u-\chi\|_{1}\right) \leqslant C h^{s}\|u\|_{s}, \quad \forall u \in \mathbf{H}^{s} \cap \stackrel{\circ}{\mathbf{H}}^{1}, 1 \leqslant s \leqslant r,
$$

and the inverse property

$$
\|\chi\|_{1} \leqslant C h^{-1}\|\chi\|, \quad \forall \chi \in \mathbf{S}_{h}^{r} .
$$

Consider also, with $r$ as above and for $0<\hat{h}<1$, a family $P_{\hat{h}}^{r}=P_{h}^{r}(\Omega)$ of finite-dimensional subspaces of $H^{1}$, (approximations to the pressure will be sought in $P_{\hat{h}}^{r} / R^{1}$ ), consisting of piecewise polynomials of degree $\leqslant 1$ if $r=2$ and $\leqslant r-2$ if $r>2$, defined on a (generally different from that associated with $\mathbf{S}_{h}^{r}$ ) quasiuniform partition of $\bar{\Omega}$. We require that $P_{\hat{h}}^{r}$ satisfy, for some constant $C$ independent of $\hat{h}$, the approximation property

$$
\inf _{\phi \in P_{h}^{r}}\left(|p-\phi|+\hat{h}|p-\phi|_{1}\right) \leqslant C \hat{h}^{s}|p|_{s}, \quad \forall p \in H^{s}, 1 \leqslant s \leqslant r-1,
$$

and the inverse property

$$
|\phi|_{1} \leqslant C \hat{h}^{-1}|\phi|, \quad \forall \phi \in P_{\hat{h}}^{r} .
$$

Let now $\mathbf{H}=\stackrel{\circ}{\mathbf{H}}^{1} \times H^{1} / R^{1}$ and $\mathbf{H}_{h, \hat{h}}=\mathbf{S}_{h}^{r} \times P_{\hat{h}}^{r} / R^{1}$. On $\mathbf{H} \times \mathbf{H}$ define the bilinear form

$$
B([u, p],[v, q])=\nu a(u, v)+(u, \operatorname{grad} q)+(v, \operatorname{grad} p)
$$

using which we can define, if $h / \hat{h}$ is sufficiently small, a suitable projection of the solution $[u, p]$ of (1.1) onto $\mathbf{H}_{h, \hat{h}}$. (See Section 2 for a statement of the relevant results, which are special cases of more general results of [2]. However, for the convenience of the reader we include their proofs in Section 4.) This projection will be suitably close to $[u, p]$ provided $\beta^{\prime} \leqslant h / \hat{h} \leqslant \beta$ holds for some constant $\beta$, sufficiently small, and any $0<\beta^{\prime} \leqslant \beta$. (All subsequent results will hold provided this condition on $h / \hat{h}$ holds.)

We now consider the trilinear form

$$
b_{1}(u, v, w)=\sum_{i, j=1}^{N} \int_{\Omega} u_{i} \frac{\partial v_{j}}{\partial x_{i}} w_{j} d x,
$$

which is well defined, e.g., for $u, v, w \in \stackrel{\mathbf{H}}{ }^{1},[18]$, since $N=2$ or 3 . We shall actually use the form

$$
b(u, v, w)=\frac{1}{2}\left(b_{1}(u, v, w)-b_{1}(u, w, v)\right) .
$$

It is well known [18, p. 163] that, for $u \in \stackrel{\circ}{\mathbf{H}}^{1}$ with $\operatorname{div} u=0$ in $\Omega$ and for $v, w \in \stackrel{\circ}{\mathbf{H}}^{1}$, there holds

$$
b(u, v, w)=b_{1}(u, v, w)=-b_{1}(u, w, v)
$$

We shall use on occasion several inequalities involving the trilinear form $b$; we shall list them in Section 2. We remark here that it is well known (since $N=2$ or 3; cf. 
$\left[18\right.$, p. 161]) that there exists a constant $C_{b}=C_{b}(\Omega)$ such that

$$
|b(u, v, w)| \leqslant C_{b}\|u\|_{1}\|v\|_{1}\|w\|_{1}, \quad \forall u, v, w \in \stackrel{\mathbf{H}}{ }^{1} .
$$

Using the notation introduced above it is easily seen that the solution $[u, p]$ of (1.1) satisfies the following weak formulation of (1.1)

$$
\left\{\begin{array}{l}
\left(u_{t}, v\right)+B([u, p],[v, q])+b(u, u, v)=(f, v), \quad \forall[v, q] \in \mathbf{H}, 0 \leqslant t \leqslant T, \\
u(0)=u^{0} .
\end{array}\right.
$$

Following [2] one may seek $u_{h}:[0, T] \rightarrow \mathbf{S}_{h}^{r}, p_{\hat{h}}:[0, T] \rightarrow P_{\hat{h}}^{r} / R^{1}$, satisfying the semidiscrete equations

$$
\begin{cases}\left(\frac{\partial u_{h}}{\partial t}, v\right)+B\left(\left[u_{h}, p_{\hat{h}}\right],[v, q]\right)+b\left(u_{h}, u_{h}, v\right)=(f, v), & \\ \left(u_{h}(0), v\right)=\left(u^{0}, v\right), \quad \forall v \in \mathbf{S}_{h}^{r} . & \forall[v, q] \in \mathbf{H}_{h, \hat{h}}, 0 \leqslant t \leqslant T,\end{cases}
$$

In [2] it is proved that a unique solution $\left[u_{h}, p_{\hat{h}}\right]$ of (1.12) exists for $0<t \leqslant T$ and that for $u, p$ sufficiently smooth the error estimates $\left\|u_{h}-u\right\|_{L^{\infty}\left(\mathbf{L}^{2}\right)}=O\left(h^{r}\right)$ and $\left|p_{\hat{h}}-p\right|_{L^{\infty}\left(L^{2} / R^{\prime}\right)}=O\left(h^{r-1}\right)$ hold. We shall not use the semidiscrete approximations $u_{h}, p_{\hat{h}}$ at all in this paper.

We now consider full discretizations of (1.12). In [2] a second order fully discrete scheme based on the trapezoidal rule with extrapolation for the nonlinear terms is analyzed. In [9] Girault and Raviart analyze the application of a family of two-step, second order accurate $A$-stable methods combined with extrapolation for the nonlinear terms for the time discretization of the abstract problem (no space discretization) for the Navier-Stokes equations. We point out that such two-step methods with extrapolation have been used for general nonlinear parabolic equations by Dupont, Fairweather and Johnson [7] and Zlámal [19], among others. In both [2] and [9] only partial extrapolation of the nonlinear terms is done, so that the resulting discrete schemes require the solution of a linear system at each time step whose linear operator changes at each time step. In [11] Jureidini considers the (fourth order in space) stream function formulation for the Navier-Stokes equations with $N=2$ and uses full extrapolation of the nonlinear terms in a fully discrete Crank-NicolsonGalerkin approximation.

We shall consider the application of the third order accurate three-step backward differentiation method, cf. e.g., [13], for the full discretization of (1.12). To that effect we let $k=T / J, k<1$ be the (constant) time step, where $J$ is a positive integer. Then, the method, in the context of approximating the solution $y(t)$, $0 \leqslant t \leqslant T$, of the (scalar) initial value problem $y^{\prime}(t)=F(y(t)), 0<t \leqslant T, y(0)=y_{0}$ given, becomes

$$
y^{n+3}-\frac{18}{11} y^{n+2}+\frac{9}{11} y^{n+1}-\frac{2}{11} y^{n}=\frac{6}{11} k F^{n+3}, \quad n=0,1, \ldots, J-3,
$$

where $y^{n}$ approximates $y\left(t^{n}\right), t^{n}=n k, F^{n}=F\left(y^{n}\right)$ and where, e.g., $y^{0}=y_{0}$ and $y^{1}$, $y^{2}$ have to be supplied by a single-step scheme. We shall use (1.13) with appropriate initial conditions to fully discretize (1.12). We shall modify the scheme by fully extrapolating (totally "lagging") the nonlinear term in (1.12) so that a single linear 
system with the same matrix need be solved at each time step. To this end we define the "lag" operator

$$
\Lambda y^{n+3}=3 y^{n+2}-3 y^{n+1}+y^{n}, \quad 0 \leqslant n \leqslant J-3,
$$

and note that $\Lambda y\left(t^{n+3}\right)=y\left(t^{n+3}\right)+O\left(k^{3}\right)$ for sufficiently smooth $y(t)$. We seek $\left\{\left[U^{n}, P^{n}\right]\right\}_{n=3}^{J} \in \mathbf{H}_{h, \hat{h}}$ satisfying

$$
\begin{aligned}
\left(U^{n+3}-\right. & \left.\frac{18}{11} U^{n+2}+\frac{9}{11} U^{n+1}-\frac{2}{11} U^{n}, v\right) \\
& +\frac{6}{11} k B\left(\left[U^{n+3}, P^{n+3}\right],[v, q]\right)+\frac{6}{11} k b\left(\Lambda U^{n+3}, \Lambda U^{n+3}, v\right) \\
= & \frac{6}{11} k\left(f^{n+3}, v\right), \quad \forall[v, q] \in \mathbf{H}_{h, \hat{h}}, 0 \leqslant n \leqslant J-3,
\end{aligned}
$$

where $U^{0}, U^{1}, U^{2}$ will be functions in $\mathbf{S}_{h}^{r}$ that will be appropriately chosen below. It will be shown in Section 2 that for each $n, 0 \leqslant n \leqslant J-3$, there exists a unique pair $\left[U^{n+3}, P^{n+3}\right] \in \mathbf{H}_{h, \hat{h}}$ satisfying (1.15) (given $U^{j} \in \mathbf{S}_{h}^{r}, j=n, n+1, n+2$ ), which is found as solution of a linear system whose matrix corresponds to the bilinear form, defined on $\left(\mathbf{H}_{h, \hat{h}}\right)^{2}$,

$$
A([U, P],[v, q])=(U, v)+\frac{6}{11} k B([U, P],[v, q]) .
$$

In order to describe our convergence results we introduce some more notation. In what follows $C$ will denote a generic constant independent of $k, h, \hat{h}, u, p, T, u^{0}$, or $f ; c$ will denote constants independent of $k, h$, and $\hat{h}$ but depending on $u^{0}, f, u$, and $p$. The constants $c$ will not depend explicitly on $T$; they may depend implicitly on $T$ though, through $L^{\alpha}(0, T ; X)$-norms of $u, p$, or $f$. We shall suppose that the solution $[u, p]$ of $(1.1)$ is smooth enough so that all constants $c$ below exist and are finite.

In Section 2 we analyze the convergence of the scheme (1.15), under the assumption that the initial values $U^{j}, j=0,1,2$, in $\mathbf{S}_{h}^{r}$ satisfy $\left\|U^{j}-u^{j}\right\| \leqslant c\left(k^{3}+h^{r}\right)$, $j=0,1,2$, where we put $u^{n}=u\left(t^{n}\right)$ for the solution of (1.1). Specifically, assuming that $k h^{-4 / 7} \leqslant \alpha$, for any positive constant $\alpha$, and that $k$ and $h$ are sufficiently small in the sense that $c\left(k^{3}+h^{r-1}\right) \leqslant \nu$ (condition (2.42)) and that

$$
c(1+T)\left(\alpha^{6} h^{10 / 7}+h^{2 r-2}\right) \leqslant \nu
$$

(condition (2.58)), then, if

$$
\|u\|_{L^{\infty}\left(\mathbf{H}^{\prime}\right)} \leqslant C \nu
$$

holds, where $C$ is a constant depending on $\Omega$ only-taken in the proof equal to $C_{a} / 660 C_{b}$ where $C_{a}$ and $C_{b}$ have been defined by (1.3) and (1.11), respectively-we prove in Theorem 2.1 that $\left\|U^{n}-u^{n}\right\| \leqslant c(1+T)^{1 / 2}\left(k^{3}+h^{r}\right), 0 \leqslant n \leqslant J$, where $c$ depends of course on $\alpha$ too.

A condition of the form (1.17) on the solution of (1.1) is needed for the proof of uniqueness and regularity of the Navier-Stokes equations for $N=3[18$, p. 303 et seq.], but is not necessary for $N=2$. However, our proof does not distinguish between the two- and the three-dimensional case. In Section 4 we show, following [18], that (1.17) holds provided $\nu$ is sufficiently large and/or the data $u^{0}, f$ are sufficiently small in appropriate function spaces. In Theorem 2.2 we give an alternative result of convergence according to which if (1.17) is not imposed, then a 
strengthening of condition (2.58) to a condition of the type $c e^{c T}\left(\alpha^{6} h^{10 / 7}+h^{2 r-2}\right) \leqslant \nu$ (condition (2.78)) will give (with the other hypotheses of Theorem 2.1 remaining unchanged) the error estimate $\left\|U^{n}-u^{n}\right\| \leqslant c e^{c T}\left(k^{3}+h^{r}\right), 0 \leqslant n \leqslant J$. For the pressure we obtain (cf. Proposition 2.3 and Theorem 2.2) the estimate

$$
\left|P^{n}-p\left(t^{n}\right)\right|_{L^{2} / R^{1}}=O\left(k^{2}+k^{-1} h^{r}+h^{r-1}\right), \quad 3 \leqslant n \leqslant J
$$

with no additional hypotheses.

In Section 3 we address the problem of choosing the initial conditions $U^{j}, j=0,1$, 2 , in $\mathbf{S}_{h}^{r}$ so that $\left\|U^{j}-u^{j}\right\|=O\left(k^{3}+h^{r}\right)$. Moreover we ask that their computation involve again only solving linear systems with the matrix corresponding to the bilinear form $A$ defined by (1.16). What complicates matters somewhat is that $p(x, 0)$ is not given as part of the initial data for $(1.1)$-if that were the case, $(1.1)$ would have been overspecified.

We first compute $U^{0}$ (and a pressure $P^{0}$ not to be used in sequel), i.e., a pair $\left[U^{0}, P^{0}\right] \in \mathbf{H}_{h, \hat{h}}$ by

$$
\begin{aligned}
\left(U^{0}, v\right)+\frac{6}{11} k B & \left(\left[U^{0}, P^{0}\right],[v, q]\right) \\
& =\left(u^{0}, v\right)+\frac{6}{11} k B\left(\left[u^{0}, 0\right],[v, q]\right), \quad \forall[v, q] \in \mathbf{H}_{h, \hat{h}} .
\end{aligned}
$$

We then obtain an intermediate value $\tilde{U}^{6 / 11}$ by the application of the single-step backward Euler method, i.e., by finding $\left[\tilde{U}^{6 / 11}, \tilde{P}^{6 / 11}\right] \in \mathbf{H}_{h, \hat{h}}$ by solving

$$
\begin{aligned}
\left(\tilde{U}^{6 / 11}-U^{0}, v\right) & +\frac{6}{11} k B\left(\left[\tilde{U}^{6 / 11}, \tilde{P}^{6 / 11}\right],[v, q]\right) \\
+ & \frac{6}{11} k b\left(u^{0}, u^{0}, v\right)=\frac{6}{11} k\left(f^{6 / 11}, v\right), \quad \forall[v, q] \in \mathbf{H}_{h, \hat{h}},
\end{aligned}
$$

where by $f^{\sigma}$ we mean of course $f\left(\cdot, t^{\sigma}\right)=f(\cdot, \sigma k)$. Using $\tilde{U}^{6 / 11}$, we compute $\left[\hat{U}_{1}, \hat{P}_{1}\right] \in \mathbf{H}_{h, \hat{h}}$ by one application of the trapezoidal method as the solution of

$$
\begin{array}{r}
\left(\hat{U}_{1}-U^{0}, v\right)+\frac{6}{11} k B\left(\left[\hat{U}_{1}, \hat{P}_{1}\right],[v, q]\right)+\frac{3}{11} k b\left(u^{0}, u^{0}, v\right) \\
+\frac{3}{11} k b\left(2 \tilde{U}^{6 / 11}-u^{0}, 2 \tilde{U}^{6 / 11}-u^{0}, v\right)=\frac{3 k}{11}\left(f^{12 / 11}+f^{0}, v\right), \\
\forall[v, q] \in \mathbf{H}_{h, \hat{h}},
\end{array}
$$

and then obtain a corrected $\left[\hat{U}_{2}, \hat{P}_{2}\right] \in \mathbf{H}_{h, \hat{h}}$ by

$$
\begin{aligned}
&\left(\hat{U}_{2}-U^{0}, v\right)+ \frac{6}{11} k B\left(\left[\hat{U}_{2}, \hat{P}_{2}\right],[v, q]\right)+\frac{3}{11} k b\left(u^{0}, u^{0}, v\right) \\
&+\frac{3}{11} k b\left(2 \hat{U}_{1}-u^{0}, 2 \hat{U}_{1}-u^{0}, v\right)=\frac{3}{11} k\left(f^{12 / 11}+f^{0}, v\right), \\
& \forall[v, q] \in \mathbf{H}_{h, \hat{h}},
\end{aligned}
$$

i.e., by one more application of the trapezoidal rule using $\hat{U}_{1}$ for the evaluation of one part of the nonlinear term. We let then

$$
U^{12 / 11}=2 \hat{U}_{2}-U^{0} \in \mathbf{S}_{h}^{r}
$$


and compute $\left[\hat{U}_{3}, P_{3}\right] \in \mathbf{H}_{h, \hat{h}}$ by

$$
\begin{aligned}
\left(\hat{U}_{3}-\right. & \left.U^{12 / 11}, v\right)+\frac{6}{11} k B\left(\left[\hat{U}_{3}, \hat{P}_{3}\right],[v, q]\right) \\
& +\frac{3}{11} k b\left(U^{12 / 11}, U^{12 / 11}, v\right)+\frac{3}{11} k b\left(2 U^{12 / 11}-u^{0}, 2 U^{12 / 11}-u^{0}, v\right) \\
& =\frac{3}{11} k\left(f^{24 / 11}+f^{12 / 11}, v\right), \quad \forall[v, q] \in \mathbf{H}_{h, \hat{h}},
\end{aligned}
$$

letting subsequently

$$
U^{24 / 11}=2 \hat{U}_{3}-U^{12 / 11} \in \mathbf{S}_{h}^{r} .
$$

It will be proved in Section 4 that $U^{0}$ is an $O\left(h^{r}\right)$ approximation (in $\mathbf{L}^{2}$ ) to $u^{0}$ and that, under the hypothesis that $k h^{-2 / 3} \leqslant \alpha$, for any positive constant $\alpha, U^{12 / 11}$, $U^{24 / 11}$ are $O\left(k^{3}+h^{r}\right)$ approximations in the $\mathbf{L}^{2}$-sense to $u^{12 / 11}, u^{24 / 11}$, respectively. Hence, finally, if we define $U^{1}, U^{2} \in \mathbf{S}_{h}^{r}$ by polynomial interpolation as

$$
\begin{aligned}
& U^{1}=\left(13 U^{0}+286 U^{12 / 11}-11 U^{24 / 11}\right) / 288, \\
& U^{2}=\left(-5 U^{0}+22 U^{12 / 11}+55 U^{24 / 11}\right) / 72,
\end{aligned}
$$

we prove in Theorem 3.1 that (for $\left.k h^{-2 / 3} \leqslant \alpha\right)\left\|U^{j}-u^{j}\right\|=O\left(k^{3}+h^{r}\right), j=0,1,2$. We note that a total of five linear systems with the matrix corresponding to the bilinear form $A$ need be solved for the determination of $U^{j}, j=0,1,2$.

We close the paper with a section of remarks in which, in addition to giving sufficient conditions for (1.17) to hold and providing the proofs of Propositions 2.1 and 2.2 , we outline the algorithm for an analogous fully discrete method that uses the two-step, second order accurate backward differentiation method and state without proof an analogous optimal rate convergence theorem.

In [14], M.-N. Le Roux considers the application of strongly $A(\theta)$-stable multistep methods (the third order backward differentiation method is strongly $A(\theta)$-stable with $\theta \cong 88^{\circ}$, cf. [13]) for the time discretization of the abstract Navier-Stokes problem (no space discretization) in two dimensions and gives an optimal rate of convergence estimate under a condition of the type (1.17) if $\theta<\pi / 2$. However, only partial extrapolation of the nonlinearity is employed so that the linear operator to be inverted at each step changes from step to step.

Backward differentiation methods of order up to six have been analyzed by Bramble and Sammon, cf., e.g., [3] for an announcement of the results, for the efficient full discretizations of Galerkin-type methods for general quasilinear parabolic equations for which the type of the nonlinearity necessitates the use of preconditioned iterative methods, cf. [6], [4], with a time-independent preconditioning operator leading to the solution of a number of linear systems with the same matrix at each step. In the Navier-Stokes case, due to the "semilinear" nature of the problem "linearization" may be achieved by totally "lagging" the nonlinear term and then solving one linear system per step, corresponding to the time-independent part of the Navier-Stokes operator.

2. Time Stepping With the Three-Step Backward Differentiation Method. In this section we analyze the convergence of the scheme (1.15) under the assumption that $U^{j}$ are optimally close in $\mathbf{L}^{2}$ to the exact values $u^{j}$ for $j=0,1,2$. First we state two 
results of [2] concerning the bilinear form $B$ defined by (1.8). These results, which appear here appropriately simplified for our purposes, will be used repeatedly. For the convenience of the reader we shall provide their proofs in Section 4 (cf. Remark 4.3). We consider

$$
\|[u, p]\|=\left(\|u\|_{1}^{2}+|p|_{L^{2} / R^{1}}^{2}\right)^{1 / 2}
$$

which is a norm on $\mathbf{H} \times \mathbf{H}$. We then have

Proposition 2.1. There exists a constant $C=C(N)$ such that for $C_{1}=C \max (\nu, 1)$ we have

$$
|B([u, p],[v, q])| \leqslant C_{1}\|[u, p]\| \mid\|[v, q]\|, \quad \forall[u, p],[v, q] \in \mathbf{H} .
$$

Moreover, there exists a positive constant $\beta$, independent of $\nu, h, \hat{h}$, such that, for $h / \hat{h} \leqslant \beta$, there exists a constant $C_{2}=C_{2}(\beta, \nu, \Omega)$ such that

$$
\sup _{\substack{[v, q] \in \mathbf{H}_{h, \hat{h}} \\\|[v, q]\| \leqslant \leqslant 1}}|B([u, p],[v, q])| \geqslant C_{2}\|[u, p]\|, \quad \forall[u, p] \in \mathbf{H}_{h, \hat{h}}
$$

In addition the following projection result holds.

Proposition 2.2. Let $[u, p]$ be the solution of (1.1), and suppose that, for $j=0,1$ and for some $1 \leqslant \alpha \leqslant \infty$,

$$
\left[\frac{\partial^{j} u}{\partial t^{j}}, \frac{\partial^{j} p}{\partial t^{j}}\right] \in L^{\alpha}\left(\left(\stackrel{\mathbf{H}}{ }^{1} \cap \mathbf{H}^{s}\right) \times H^{s-1} / R^{1}\right), \quad 2 \leqslant s \leqslant r .
$$

Suppose that $h / \hat{h} \leqslant \beta$ as in Proposition 2.1 and that, for some positive constant $\beta^{\prime}$, there holds that $\beta^{\prime} \leqslant h / \hat{h}$. Then, there exists a unique pair $[\omega, \pi] \in L^{\alpha}\left(\mathbf{H}_{h, \hat{h}}\right)-$ henceforth referred to as the "B-projection" of $[u, p]$ onto $\mathbf{H}_{h, \hat{h}}$-satisfying

$$
\begin{aligned}
B([\omega(t), \pi(t)],[v, q])=B([u(t), p(t)],[v, q]), & \\
\forall[v, q] & \in \mathbf{H}_{h, \hat{h}}, 0 \leqslant t \leqslant T .
\end{aligned}
$$

Moreover, the following error estimates hold for some constant $C$, independent of $h, \hat{h}$, $u, p$, and $T$ :

$$
\begin{aligned}
& \left\|\frac{\partial^{j}}{\partial t^{j}}(u-\omega)\right\|_{L^{\alpha}\left(\mathbf{H}^{\mu}\right)} \leqslant C h^{s-\mu}\left(\left\|\frac{\partial^{j} u}{\partial t^{j}}\right\|_{L^{\alpha}\left(\mathbf{H}^{s}\right)}+\left|\frac{\partial^{j} p}{\partial t^{j}}\right|_{L^{\alpha}\left(H^{s-1} / R^{1}\right)}\right), \\
& 2 \leqslant s \leqslant r, \mu, j=0,1 . \\
& |p-\pi|_{L^{\alpha}\left(L^{2} / R^{1}\right)} \leqslant C h^{s-1}\left(\|u\|_{L^{\alpha}\left(\mathbf{H}^{s}\right)}+|p|_{L^{\alpha}\left(H^{s-1} / R^{1}\right)}\right), \quad 2 \leqslant s \leqslant r . \quad \square
\end{aligned}
$$

Henceforth we shall always assume that $\beta^{\prime} \leqslant h / \hat{h} \leqslant \beta$, so that the conclusions of Propositions 2.1 and 2.2 hold, under the appropriate smoothness assumptions on $u$ and $p$.

It is easy now to see that, for each $0 \leqslant n \leqslant J-3$, given $U^{j} \in \mathbf{S}_{h}^{r}, j=n, n+1$, $n+2$, a unique solution $\left[U^{n+3}, P^{n+3}\right] \in \mathbf{H}_{h, \hat{h}}$ of (1.15) exists. For, assuming that there exists $[U, P] \in \mathbf{H}_{h, \hat{h}}$ satisfying

$$
A([U, P],[v, q])=0, \quad \forall[v, q] \in \mathbf{H}_{h, \hat{h}},
$$

(where the bilinear form $A$ has been defined by (1.16)) and choosing $[v, q]=[U,-P]$ in (2.6), we obtain by (1.8) that $U=0$. It follows by (2.6) that $(v, \operatorname{grad} P)=0$ $\forall v \in \mathbf{S}_{h}^{r}$. Putting now $u=0, p=P$ in (2.2) we see that $P=0$ in $P_{h}^{r} / R^{l}$. Hence (2.6) 
has only the trivial solution, a fact that establishes the existence and uniqueness of the solution $\left[U^{n+3}, P^{n+3}\right], 0 \leqslant n \leqslant J-3$, of (1.15).

We shall henceforth denote by $\tilde{\mathbf{S}}=\tilde{\mathbf{S}}(r, h, \hat{h})$ the subspace of $\mathbf{S}_{h}^{r}$ consisting of all functions $v \in \mathbf{S}_{h}^{r}$ for which $(v, \operatorname{grad} q)=0$ holds for all $q \in P_{\hat{h}}^{r}$. Clearly $\tilde{\mathbf{S}}$ is not empty under the hypotheses of Propositions 2.1 and 2.2. For example, putting $v=0$ in (2.3) and using the fact that $\operatorname{div} u(t)=0$, we see that $\omega(t) \in \tilde{\mathbf{S}}$ for $t \in[0, T]$.

Before proceeding to the error estimates we list some more inequalities associated with the trilinear form $b(u, v, w)$, defined by (1.9), that will be repeatedly used in sequel along with (1.11). It follows easily from (1.9) that there exists a constant $C=C(N)$ such that

$$
\begin{aligned}
& |b(u, v, w)| \leqslant C\left(\|u\|\|v\|_{1, \infty}\|w\|+\|u\|\|v\|_{\infty}\|w\|_{1}\right), \\
& \qquad \forall u \in \mathbf{L}^{2}, v \in \mathbf{W}^{1, \infty}, w \in \mathbf{H}^{1} .
\end{aligned}
$$

On the other hand, it follows from (1.10) that there exists a constant $C=C(N)$ such that for $u \in \stackrel{\mathbf{H}}{ }^{1} \cap \mathbf{L}^{\infty}$, such that $\operatorname{div} u=0$ in $\Omega$ and for $v, w \in \stackrel{\mathbf{H}}{ }^{1}$,

$$
|b(u, v, w)| \leqslant C\|u\|_{\infty}\|v\|\|w\|_{1}
$$

and

$$
|b(u, v, w)| \leqslant C\|u\|_{\infty}\|v\|_{1}\|w\|
$$

hold. In addition, for $u \in \stackrel{\mathrm{H}}{ }^{1}$ with $\operatorname{div} u=0$ in $\Omega$ and for $v \in \stackrel{\circ}{\mathbf{H}}^{1} \cap \mathbf{W}^{1, \infty}, w \in \stackrel{\circ}{\mathbf{H}}^{1}$ we have that

$$
|b(u, v, w)| \leqslant C\|u\|\|v\|_{1, \infty}\|w\|
$$

and if $u \in \stackrel{\circ}{\mathbf{H}}^{1}$ with $\operatorname{div} u=0$ in $\Omega$ and if $v \in \stackrel{\circ}{\mathbf{H}}^{1} \cap \mathbf{L}^{\infty}, w \in \stackrel{\circ}{\mathbf{H}}^{1}$,

$$
|b(u, v, w)| \leqslant C\|u\|\|v\|_{\infty}\|w\|_{1} .
$$

Let now $[\omega(t), \pi(t)] \in \mathbf{H}_{h, \hat{h}}, 0 \leqslant t \leqslant T$, be the $B$-projection, defined by (2.3), of the solution $[u(t), p(t)]$ of $(1.1)$, and put $y^{n}=y\left(t^{n}\right), 0 \leqslant n \leqslant J$, for a function $y$ defined in $[0, T]$. Define $\zeta^{n}=U^{n}-\omega^{n}, 0 \leqslant n \leqslant J, e^{n}=P^{n}-\pi^{n}, 3 \leqslant n \leqslant J, \eta(t)$ $=u(t)-\omega(t), 0 \leqslant t \leqslant T$. Then, using (2.3) and (1.15), we easily obtain

$$
\begin{array}{r}
\left(\zeta^{n+3}-\frac{18}{11} \zeta^{n+2}+\frac{9}{11} \zeta^{n+1}-\frac{2}{11} \zeta^{n}, v\right)+\frac{6}{11} k B\left(\left[\zeta^{n+3}, e^{n+3}\right],[v, q]\right) \\
=\left(\rho^{n+3}+\delta^{n+3}, v\right)+\frac{6}{11} k\left(b\left(u^{n+3}, u^{n+3}, v\right)-b\left(\Lambda U^{n+3}, \Lambda U^{n+3}, v\right)\right) \\
\forall[v, q] \in \mathbf{H}_{h, \hat{h}}, 0 \leqslant n \leqslant J-3
\end{array}
$$

where

$$
\begin{aligned}
\rho^{n+3}=\frac{6}{11} k u_{t}^{n+3}-\left(u^{n+3}-\frac{18}{11} u^{n+2}+\frac{9}{11} u^{n+1}-\frac{2}{11} u^{n}\right) & , \\
0 & \leqslant n \leqslant J-3,
\end{aligned}
$$

and

$$
\delta^{n+3}=\eta^{n+3}-\frac{18}{11} \eta^{n+2}+\frac{9}{11} \eta^{n+1}-\frac{2}{11} \eta^{n}, \quad 0 \leqslant n \leqslant J-3
$$


We look first at the nonlinear terms in the right-hand side of (2.10), which we write as

$$
\begin{aligned}
& b\left(u^{n+3}, u^{n+3}, v\right)-b\left(\Lambda U^{n+3}, \Lambda U^{n+3}, v\right) \\
&= b\left(u^{n+3}, u^{n+3}-\Lambda u^{n+3}, v\right)+b\left(u^{n+3}, \Lambda \eta^{n+3}, v\right) \\
&-b\left(u^{n+3}, \Lambda \zeta^{n+3}, v\right)+b\left(u^{n+3}-\Lambda u^{n+3}, \Lambda \omega^{n+3}, v\right) \\
&+b\left(u^{n+3}-\Lambda u^{n+3}, \Lambda \zeta^{n+3}, v\right)+b\left(\Lambda \eta^{n+3}, \Lambda u^{n+3}, v\right) \\
&-b\left(\Lambda \eta^{n+3}, \Lambda \eta^{n+3}, v\right)+b\left(\Lambda \zeta^{n+3}, \Lambda \eta^{n+3}, v\right) \\
&-b\left(\Lambda \zeta^{n+3}, u^{n+3}, v\right)+b\left(\Lambda \zeta^{n+3}, u^{n+3}-\Lambda u^{n+3}, v\right) \\
&+b\left(\Lambda \eta^{n+3}, \Lambda \zeta^{n+3}, v\right)-b\left(\Lambda \zeta^{n+3}, \Lambda \zeta^{n+3}, v\right), \quad 0 \leqslant n \leqslant J-3
\end{aligned}
$$

We now estimate the individual terms in the right-hand side of (2.12). Since $u$ is the solution of (1.1), $\operatorname{div} u=0$ in $\Omega$ and hence (2.8) gives

$$
\begin{aligned}
& \left|b\left(u^{n+3}, u^{n+3}-\Lambda u^{n+3}, v\right)\right| \\
& \quad \leqslant C\left\|u^{n+3}\right\|_{\infty}\left\|u^{n+3}-\Lambda u^{n+3}\right\|\|v\|_{1} \\
& \quad \leqslant C k^{3}\|u\|_{L^{\infty}\left(\mathbf{L}^{\infty}\right)}\left\|\frac{\partial^{3} u}{\partial t^{3}}\right\|_{L^{\infty}\left(\mathbf{L}^{2}\right)}\|v\|_{1}, \quad 0 \leqslant n \leqslant J-3 .
\end{aligned}
$$

Using again (2.8) and also (2.4), we obtain

$$
\begin{aligned}
& \left|b\left(u^{n+3}, \Lambda \eta^{n+3}, v\right)\right| \leqslant C\left\|u^{n+3}\right\|_{\infty}\left\|\Lambda \eta^{n+3}\right\|\|v\|_{1} \\
& \quad \leqslant C h^{r}\|u\|_{L^{\infty}\left(\mathbf{L}^{\infty}\right)}\left(\|u\|_{L^{\infty}\left(\mathbf{H}^{r}\right)}+|p|_{L^{\infty}\left(H^{r-1} / R^{\prime}\right)}\right)\|v\|_{1}, \quad 0 \leqslant n \leqslant J-3 .
\end{aligned}
$$

For the purposes of Theorem 2.1 we shall estimate the third term of the right-hand side of (2.12) in two different ways depending on $n$. First, using (1.11), we have

$$
\begin{aligned}
\left|b\left(u^{n+3}, \Lambda \zeta^{n+3}, v\right)\right| & \leqslant C_{b}\left\|u^{n+3}\right\|_{1}\left\|\Lambda \zeta^{n+3}\right\|_{1}\|v\|_{1} \\
& \leqslant C_{b}\|u\|_{L^{\infty}\left(\mathbf{H}^{\prime}\right)}\left\|\Lambda \zeta^{n+3}\right\|_{1}\|v\|_{1}, \quad 3 \leqslant n \leqslant J-3
\end{aligned}
$$

In addition, using (2.8),

$$
\begin{aligned}
\left|b\left(u^{n+3}, \Lambda \zeta^{n+3}, v\right)\right| & \leqslant C\left\|u^{n+3}\right\|_{\infty}\left\|\Lambda \zeta^{n+3}\right\|\|v\|_{1} \\
& \leqslant C\|u\|_{L^{\infty}\left(\mathbf{L}^{\infty}\right)}\left\|\Lambda \zeta^{n+3}\right\|\|v\|_{1}, \quad n=0,1,2 .
\end{aligned}
$$

Again (2.8) gives

$$
\begin{aligned}
\left|b\left(u^{n+3}-\Lambda u^{n+3}, \Lambda \omega^{n+3}, v\right)\right| \leqslant C\left\|u^{n+3}-\Lambda u^{n+3}\right\|_{\infty}\left\|\Lambda \omega^{n+3}\right\|\|v\|_{1}, \\
0 \leqslant n \leqslant J-3 .
\end{aligned}
$$

To estimate $\left\|\Lambda \omega^{n+3}\right\|$ we recall that $\omega(t) \in \tilde{\mathbf{S}}$. Hence, putting $v=\omega$ in (2.3), we see that

$$
\begin{aligned}
\nu a(\omega, \omega) & =\nu a(u, \omega)+(\omega, \operatorname{grad} p)=\nu a(u, \omega)+\langle\operatorname{div} \omega, p+C\rangle \\
& \leqslant C\left(\|u\|_{1}+|p|_{L^{2} / R^{1}}\right)\|\omega\|_{1} .
\end{aligned}
$$

It follows that

$$
\|\omega\|_{L^{\infty}\left(\mathbf{L}^{2}\right)} \leqslant\|\omega\|_{L^{\infty}\left(\mathbf{H}^{\prime}\right)} \leqslant C\left(\|u\|_{L^{\infty}\left(\mathbf{H}^{1}\right)}+|p|_{L^{\infty}\left(L^{2} / R^{1}\right)}\right) .
$$


Hence we see that

$$
\begin{aligned}
& \left|b\left(u^{n+3}-\Lambda u^{n+3}, \Lambda \omega^{n+3}, v\right)\right| \\
& \quad \leqslant C k^{3}\|v\|_{1}\left\|\frac{\partial^{3} u}{\partial t^{3}}\right\|_{L^{\infty}\left(L^{\infty}\right)}\left(\|u\|_{L^{\infty}\left(\mathbf{H}^{1}\right)}+|p|_{L^{\infty}\left(L^{2} / R^{1}\right)}\right), \quad 0 \leqslant n \leqslant J-3 .
\end{aligned}
$$

Applying (1.11), we now obtain

$$
\begin{aligned}
\mid b\left(u^{n+3}-\Lambda u^{n+3},\right. & \left.\Lambda \zeta^{n+3}, v\right) \mid \leqslant C_{b}\left\|u^{n+3}-\Lambda u^{n+3}\right\|_{1}\left\|\Lambda \zeta^{n+3}\right\|_{1}\|v\|_{1} \\
& \leqslant C k^{3}\left\|\frac{\partial^{3} u}{\partial t^{3}}\right\|_{L^{\infty}\left(\mathbf{H}^{\prime}\right)}\left\|\Lambda \zeta^{n+3}\right\|_{1}\|v\|_{1}, \quad 0 \leqslant n \leqslant J-3
\end{aligned}
$$

From (2.7), (2.4) it follows that

$$
\begin{gathered}
\left|b\left(\Lambda \eta^{n+3}, \Lambda u^{n+3}, v\right)\right| \leqslant C\left(\left\|\Lambda \eta^{n+3}\right\|\left\|\Lambda u^{n+3}\right\|_{1, \infty}\|v\|\right. \\
\left.+\left\|\Lambda \eta^{n+3}\right\|\left\|\Lambda u^{n+3}\right\|_{\infty}\|v\|_{1}\right) \\
\leqslant C h^{r}\|u\|_{L^{\infty}\left(\mathbf{W}^{1, \infty}\right)}\left(\|u\|_{L^{\infty}\left(\mathbf{H}^{r}\right)}+|p|_{L^{\infty}\left(H^{r-1} / R^{1}\right)}\right) \cdot\|v\|_{1}, \\
0 \leqslant n \leqslant J-3 .
\end{gathered}
$$

Using now (1.11), (2.4) and the facts that $h<1, r \geqslant 2$, we see that

$$
\begin{aligned}
& \left|b\left(\Lambda \eta^{n+3}, \Lambda \eta^{n+3}, v\right)\right| \leqslant C_{b}\left\|\Lambda \eta^{n+3}\right\|_{1}^{2}\|v\|_{1} \\
& \quad \leqslant C h^{r}\left(\|u\|_{L^{\infty}\left(\mathbf{H}^{r}\right)}+|p|_{L^{\infty}\left(H^{r-1} / R^{1}\right)}\right)^{2}\|v\|_{1}, \quad 0 \leqslant n \leqslant J-3 .
\end{aligned}
$$

Again, (1.11) and (2.4) give

$$
\begin{aligned}
& \left|b\left(\Lambda \zeta^{n+3}, \Lambda \eta^{n+3}, v\right)\right| \leqslant C_{b}\left\|\Lambda \zeta^{n+3}\right\|_{1}\left\|\Lambda \eta^{n+3}\right\|_{1}\|v\|_{1} \\
& \quad \leqslant C h^{r-1}\left(\|u\|_{L^{\infty}\left(\mathbf{H}^{\prime}\right)}+|p|_{L^{\infty}\left(H^{r-1} / R^{1}\right)}\right)\left\|\Lambda \zeta^{n+3}\right\|_{1}\|v\|_{1}, \quad 0 \leqslant n \leqslant J-3 .
\end{aligned}
$$

Using (1.11), we obtain

(2.21) $\left|b\left(\Lambda \zeta^{n+3}, u^{n+3}, v\right)\right| \leqslant C_{b}\|u\|_{L^{\infty}\left(\mathbf{H}^{1}\right)}\left\|\Lambda \zeta^{n+3}\right\|_{1}\|v\|_{1}, \quad 3 \leqslant n \leqslant J-3$,

while (2.7) gives

$$
\begin{aligned}
\left|b\left(\Lambda \zeta^{n+3}, u^{n+3}, v\right)\right| & \leqslant C\left(\left\|\Lambda \zeta^{n+3}\right\|\left\|u^{n+3}\right\|_{1, \infty}\|v\|+\left\|\Lambda \zeta^{n+3}\right\|\left\|u^{n+3}\right\|_{\infty}\|v\|_{1}\right) \\
& \leqslant C\|u\|_{L^{\infty}\left(\mathbf{W}^{1, \infty}\right)}\left\|\Lambda \zeta^{n+3}\right\|\|v\|_{1}, \quad n=0,1,2
\end{aligned}
$$

From (1.11) we obtain

$$
\begin{aligned}
\mid b\left(\Lambda \zeta^{n+3}, u^{n+3}-\right. & \left.\Lambda u^{n+3}, v\right) \mid \leqslant C_{b}\left\|\Lambda \zeta^{n+3}\right\|_{1}\left\|u^{n+3}-\Lambda u^{n+3}\right\|_{1}\|v\|_{1} \\
& \leqslant C k^{3}\left\|\frac{\partial^{3} u}{\partial t^{3}}\right\|_{L^{\infty}\left(\mathbf{H}^{\prime}\right)}\left\|\Lambda \zeta^{n+3}\right\|_{1}\|v\|_{1}, \quad 0 \leqslant n \leqslant J-3 .
\end{aligned}
$$

By (1.11), (2.4) we see that

$$
\begin{aligned}
& \left|b\left(\Lambda \eta^{n+3}, \Lambda \zeta^{n+3}, v\right)\right| \leqslant C_{b}\left\|\Lambda \eta^{n+3}\right\|_{1}\left\|\Lambda \zeta^{n+3}\right\|_{1}\|v\|_{1} \\
& \quad \leqslant C h^{r-1}\left(\|u\|_{L^{\infty}\left(\mathbf{H}^{r}\right)}+|p|_{L^{\infty}\left(H^{r-1} / R^{1}\right)}\right)\left\|\Lambda \zeta^{n+3}\right\|_{1}\|v\|_{1}, \quad 0 \leqslant n \leqslant J-3 .
\end{aligned}
$$

Finally, (1.11) gives

$$
\left|b\left(\Lambda \zeta^{n+3}, \Lambda \zeta^{n+3}, v\right)\right| \leqslant C_{b}\left\|\Lambda \zeta^{n+3}\right\|_{1}^{2}\|v\|_{1}, \quad 0 \leqslant n \leqslant J-3 .
$$


We return now to the estimation of $\zeta^{n}$ in (2.10). For reasons that will become evident in Section 3, where the error estimates for $\left\|U^{j}-u^{j}\right\|, j=0,1,2$, will be given, we need to estimate $\zeta^{n}, n=3,4,5$, in a different way than $\zeta^{n}, 6 \leqslant n \leqslant J$. Henceforth we shall make the hypothesis (to be verified in Section 3 for our particular initial conditions) that

$$
\left\|\zeta^{j}\right\| \leqslant c_{0}\left(k^{3}+h^{r}\right), \quad j=0,1,2,
$$

is satisfied. Here, the lower-case constant $c_{0}$ depends on $u, p$ as noted in the Introduction.

LEMMA 2.1. Let (2.25) be satisfied. Then, if we assume that

$$
k h^{-4 / 7} \leqslant \alpha
$$

for any positive constant $\alpha$ (i.e. not necessarily small), then there exists a constant $c_{1}=c_{1}(u, p, \alpha)$ such that

$$
\left\|\zeta^{\prime}\right\|^{2}+k \nu a\left(\zeta^{\prime}, \zeta^{\prime}\right) \leqslant c_{1}\left(k^{3}+h^{r}\right)^{2}, \quad l=3,4,5
$$

Proof. Putting $v=0$ in (2.10), we see that $\left(\zeta^{n+3}, \operatorname{grad} q\right)=0 \forall q \in P_{h}^{r} / R^{1}$, i.e., that $\zeta^{n+3} \in \tilde{\mathbf{S}}$, for $0 \leqslant n \leqslant J-3$. Hence the choice $v=\zeta^{n+3}$ in (2.10) gives

$$
\begin{aligned}
\left(\zeta^{n+3}-\frac{18}{11} \zeta^{n+2}+\frac{9}{11} \zeta^{n+1}-\frac{2}{11} \zeta^{n}, \zeta^{n+3}\right)+\frac{6}{11} k \nu a\left(\zeta^{n+3}, \zeta^{n+3}\right) & \\
= & \left(\rho^{n+3}+\delta^{n+3}, \zeta^{n+3}\right) \\
& +\frac{6}{11} k\left(b\left(u^{n+3}, u^{n+3}, \zeta^{n+3}\right)-b\left(\Lambda U^{n+3}, \Lambda U^{n+3}, \zeta^{n+3}\right)\right) \\
& 0 \leqslant n \leqslant J-3 .
\end{aligned}
$$

Using now the Cauchy-Schwarz inequality and the inequality $2 a b \leqslant a^{2} / \varepsilon+\varepsilon b^{2}$ for $\varepsilon>0$, we obtain, for any $\varepsilon_{1}, \varepsilon_{2}>0$,

$$
\begin{aligned}
\left(1-\varepsilon_{2} \frac{29}{22}\right) & \left\|\zeta^{n+3}\right\|^{2}+\frac{6}{11} k \nu a\left(\zeta^{n+3}, \zeta^{n+3}\right) \\
\leqslant & \frac{1}{22 \varepsilon_{2}}\left(18\left\|\zeta^{n+2}\right\|^{2}+9\left\|\zeta^{n+1}\right\|^{2}+2\left\|\zeta^{n}\right\|^{2}\right) \\
& +\frac{1}{2 \varepsilon_{1} k}\left\|\rho^{n+3}+\delta^{n+3}\right\|^{2}+\frac{k \varepsilon_{1}}{2}\left\|\zeta^{n+3}\right\|_{1}^{2} \\
& +\frac{6}{11} k\left|b\left(u^{n+3}, u^{n+3}, \zeta^{n+3}\right)-b\left(\Lambda U^{n+3}, \Lambda U^{n+3}, \zeta^{n+3}\right)\right|, \quad n=0,1,2 .
\end{aligned}
$$

Then, using the second inequality of (1.3) and choosing $\varepsilon_{2}=11 / 29, \varepsilon_{1}=\nu C_{a} / 11$, we obtain

$$
\begin{aligned}
\left\|\zeta^{n+3}\right\|^{2}+ & k \nu a\left(\zeta^{n+3}, \zeta^{n+3}\right) \\
\leqslant & C\left(\left\|\zeta^{n+2}\right\|^{2}+\left\|\zeta^{n+1}\right\|^{2}+\left\|\zeta^{n}\right\|^{2}\right)+C k^{-1}\left\|\rho^{n+3}+\delta^{n+3}\right\|^{2} \\
& +\frac{12 k}{11}\left|b\left(u^{n+3}, u^{n+3}, \zeta^{n+3}\right)-b\left(\Lambda U^{n+3}, \Lambda U^{n+3}, \zeta^{n+3}\right)\right| \\
n & =0,1,2
\end{aligned}
$$


Now, (2.11) and $\left(2.11^{\prime}\right),(2.4)$ and the consistency of (1.13) give

$$
\left\|\rho^{n+3}\right\| \leqslant C k^{4}\left\|\frac{\partial^{4} u}{\partial t^{4}}\right\|_{L^{\infty}\left(\mathbf{L}^{2}\right)}, \quad 0 \leqslant n \leqslant J-3,
$$

(2.31) $\left\|\delta^{n+3}\right\| \leqslant C k h^{r}\left(\left\|\frac{\partial u}{\partial t}\right\|_{L^{\infty}\left(\mathbf{H}^{r}\right)}+\left|\frac{\partial p}{\partial t}\right|_{L^{\infty}\left(H^{r-1} / R^{1}\right)}\right), \quad 0 \leqslant n \leqslant J-3$.

Using now (2.30), (2.31) and (2.12)-(2.14), (2.15'), (2.16)-(2.20), (2.21'), (2.22)(2.24) in the right-hand side of (2.29), we obtain

$$
\begin{aligned}
\left\|\zeta^{n+3}\right\|^{2}+ & k \nu a\left(\zeta^{n+3}, \zeta^{n+3}\right) \\
\leqslant & C\left(\left\|\zeta^{n+2}\right\|^{2}+\left\|\zeta^{n+1}\right\|^{2}+\left\|\zeta^{n}\right\|^{2}\right) \\
& +c k\left(k^{3}+h^{r}\right)^{2}+c k\left(k^{3}+h^{r}+\left\|\Lambda \zeta^{n+3}\right\|+k^{3}\left\|\Lambda \zeta^{n+3}\right\|_{1}\right. \\
& \left.+h^{r-1}\left\|\Lambda \zeta^{n+3}\right\|_{1}+\left\|\Lambda \zeta^{n+3}\right\|_{1}^{2}\right)\left\|\zeta^{n+3}\right\|_{1}, \quad n=0,1,2,
\end{aligned}
$$

which gives upon application of the arithmetic-geometric mean inequality with $\varepsilon>0$ that

$$
\begin{aligned}
&\left\|\zeta^{n+3}\right\|^{2}+k \nu a\left(\zeta^{n+3}, \zeta^{n+3}\right) \\
& \leqslant C\left(\left\|\zeta^{n+2}\right\|^{2}+\left\|\zeta^{n+1}\right\|^{2}+\left\|\zeta^{n}\right\|^{2}\right)+c k\left(k^{3}+h^{r}\right)^{2} \\
&+c \varepsilon^{-1}\left\{k\left(k^{3}+h^{r}\right)^{2}+k\left\|\Lambda \zeta^{n+3}\right\|^{2}+k^{7}\left\|\Lambda \zeta^{n+3}\right\|_{1}^{2}\right. \\
&\left.+k h^{2 r-2}\left\|\Lambda \zeta^{n+3}\right\|_{1}^{2}+k\left\|\Lambda \zeta^{n+3}\right\|_{1}^{4}\right\}+\varepsilon k\left\|\zeta^{n+3}\right\|_{1}^{2}, \\
& n=0,1,2 .
\end{aligned}
$$

Hence, picking, e.g., $\varepsilon=\nu C_{a} / 2$ yields

$$
\begin{aligned}
& \left\|\zeta^{n+3}\right\|^{2}+k \nu a\left(\zeta^{n+3}, \zeta^{n+3}\right) \\
& \leqslant c k\left(k^{3}+h^{r}\right)^{2} \\
& \quad+c\left\{\sum_{i=0}^{2}\left\|\zeta^{n+i}\right\|^{2}+\left(k h^{2 r-2}+k^{7}\right) \sum_{i=0}^{2}\left\|\zeta^{n+i}\right\|_{1}^{2}+k \sum_{i=0}^{2}\left\|\zeta^{n+i}\right\|_{1}^{4}\right\}, \\
& \quad n=0,1,2 .
\end{aligned}
$$

Consider first (2.32) for $n=0$. Using the inverse assumption (1.5), the hypothesis (2.25), the fact that $k, h<1$ and $r \geqslant 2$ and the hypothesis (2.26), we see that

$$
\begin{aligned}
\left(k h^{2 r-2}\right. & \left.+k^{7}\right) \sum_{i=0}^{2}\left\|\zeta^{i}\right\|_{1}^{2} \leqslant c\left(k h^{2 r-2}+k^{7}\right) h^{-2}\left(k^{3}+h^{r}\right)^{2} \\
& \leqslant c\left(k^{7} h^{2 r-4}+k h^{4 r-4}+k^{13} h^{-2}+k^{7} h^{2 r-2}\right) \leqslant c(\alpha)\left(k^{6}+h^{2 r}\right) .
\end{aligned}
$$

Moreover, (1.5), (2.26) and (2.25) give also

$$
\begin{aligned}
k \sum_{i=0}^{2}\left\|\zeta^{i}\right\|_{1}^{4} & \leqslant c k h^{-4}\left(k^{3}+h^{r}\right)^{4} \\
& \leqslant c\left(k^{13} h^{-4}+k h^{4 r-4}\right) \leqslant c(\alpha)\left(k^{6}+h^{2 r}\right) .
\end{aligned}
$$

Now, (2.25), (2.33), and (2.34) substituted in (2.32) yield

$$
\left\|\zeta^{3}\right\|^{2}+k \nu a\left(\zeta^{3}, \zeta^{3}\right) \leqslant c(\alpha)\left(k^{3}+h^{r}\right)^{2} .
$$


In particular, $\left\|\zeta^{3}\right\|=O\left(k^{3}+h^{r}\right)$, and the argument can be repeated using (2.32) for $n=1$ and then for $n=2$ to yield (2.27). Of course, this type of estimation can only be used for a small (i.e., independent of $k$ ) number of steps.

We continue now with the estimation of $\zeta^{n+3}$ for $3 \leqslant n \leqslant J-3$. As an intermediate step we prove the following

LEMMA 2.2. Let $k$ and $h$ be sufficiently small (cf. (2.42) below). Then if

$$
\|u\|_{L^{\infty}\left(\mathbf{H}^{\prime}\right)} \leqslant \tilde{C} \nu
$$

holds, where $\tilde{C}=\tilde{C}(\Omega)$ is a constant that can be determined, e.g., by (2.43) below, it follows that

$$
\begin{aligned}
& \left\|\Gamma \zeta^{n+3}\right\|^{2}-\left\|\Gamma \zeta^{n+2}\right\|^{2}+\frac{73}{121} k \nu a\left(\zeta^{n+3}, \zeta^{n+3}\right) \\
& -\frac{47}{121} k \nu a\left(\zeta^{n+2}, \zeta^{n+2}\right)-\frac{17}{121} k \nu a\left(\zeta^{n+1}, \zeta^{n+1}\right)-\frac{4 k \nu}{121} a\left(\zeta^{n}, \zeta^{n}\right) \\
& \leqslant c k\left(k^{3}+h^{r}\right)^{2}+C k\left\|\Lambda \zeta^{n+3}\right\|_{1}^{4}, \quad 3 \leqslant n \leqslant J-3,
\end{aligned}
$$

where

$$
\Gamma \zeta^{l} \equiv \zeta^{l}-\frac{7}{11} \zeta^{l-1}+\frac{2}{11} \zeta^{l-2}, \quad 2 \leqslant l \leqslant J
$$

Proof. Noting that

$$
\zeta^{n+3}-\frac{18}{11} \zeta^{n+2}+\frac{9}{11} \zeta^{n+1}-\frac{2}{11} \zeta^{n}=\Gamma \zeta^{n+3}-\Gamma \zeta^{n+2}
$$

and since $\zeta^{n+3} \in \tilde{\mathbf{S}}$ for $0 \leqslant n \leqslant J-3$, inserting $v=\Gamma \zeta^{n+3}$ in (2.10) gives

$$
\begin{aligned}
&\left\|\Gamma \zeta^{n+3}\right\|^{2}-\left(\Gamma \zeta^{n+2}, \Gamma \zeta^{n+3}\right)+\frac{6}{11} k k a\left(\zeta^{n+3}, \Gamma \zeta^{n+3}\right) \\
&=\left(\rho^{n+3}+\delta^{n+3}, \Gamma \zeta^{n+3}\right) \\
&+\frac{6}{11} k\left(b\left(u^{n+3}, u^{n+3}, \Gamma \zeta^{n+3}\right)-b\left(\Lambda U^{n+3}, \Lambda U^{n+3}, \Gamma \zeta^{n+3}\right)\right), \\
& 3 \leqslant n \leqslant J-3 .
\end{aligned}
$$

Using the Cauchy-Schwarz inequality and the arithmetic-geometric mean inequality for the last two terms of the left-hand side of the above and estimating its right-hand side using (2.12)-(2.24), we obtain

$$
\begin{aligned}
&\left\|\Gamma \zeta^{n+3}\right\|^{2}-\left\|\Gamma \zeta^{n+2}\right\|^{2}+\frac{78}{121} k \nu a\left(\zeta^{n+3}, \zeta^{n+3}\right) \\
&-\frac{42}{121} k \nu a\left(\zeta^{n+2}, \zeta^{n+2}\right)-\frac{12}{121} k \nu a\left(\zeta^{n+1}, \zeta^{n+1}\right) \\
& \leqslant 2\left\|\rho^{n+3}+\delta^{n+3}\right\|\left\|\Gamma \zeta^{n+3}\right\|_{1} \\
&+\frac{12}{11} k\left\{c\left(k^{3}+h^{r}\right)+c^{\prime}\left(k^{3}+h^{r-1}\right)\left\|\Lambda \zeta^{n+3}\right\|_{1}\right. \\
&\left.+2 C_{b}\|u\|_{L^{\infty}\left(\mathbf{H}^{1}\right)}\left\|\Lambda \zeta^{n+3}\right\|_{1}+C_{b}\left\|\Lambda \zeta^{n+3}\right\|_{1}^{2}\right\}\left\|\Gamma \zeta^{n+3}\right\|_{1}, \\
& \quad 3 \leqslant n \leqslant J-3 .
\end{aligned}
$$


where we note from (2.17), (2.20), (2.22), (2.23) that

$$
c^{\prime}=C\left(\left\|\frac{\partial^{3} u}{\partial t^{3}}\right\|_{L^{\infty}\left(\mathbf{H}^{1}\right)}+\|u\|_{L^{\infty}\left(\mathbf{H}^{r}\right)}+|p|_{L^{\infty}\left(H^{r-1} / R^{1}\right)}\right) .
$$

We now estimate some of the terms in the right-hand side of (2.38), using the weighted arithmetic-geometric mean inequality for $\varepsilon>0$ and (2.30), (2.31), (1.3) and the definition of $\Gamma \zeta^{\prime}$

$$
\begin{aligned}
\left\{2 \| \rho^{n+3}+\right. & \left.\delta^{n+3}\left\|+\frac{12}{11} c k\left(k^{3}+h^{r}\right)+\frac{12}{11} C_{b} k\right\| \Lambda \zeta^{n+3} \|_{1}^{2}\right\}\left\|\Gamma \zeta^{n+3}\right\|_{1} \\
\leqslant & c \varepsilon^{-1} k\left(k^{3}+h^{r}\right)^{2}+C \varepsilon^{-1} k\left\|\Lambda \zeta^{n+3}\right\|_{1}^{4} \\
& +\varepsilon k\left[a\left(\zeta^{n+3}, \zeta^{n+3}\right)+a\left(\zeta^{n+2}, \zeta^{n+2}\right)+a\left(\zeta^{n+1}, \zeta^{n+1}\right)\right], \\
& \quad 3 \leqslant n \leqslant J-3 .
\end{aligned}
$$

Hence, choosing, e.g., $\varepsilon=\nu / 121$, we obtain from (2.38) and the above, using again (1.3),

$$
\begin{aligned}
&\left\|\Gamma \zeta^{n+3}\right\|^{2}-\| \Gamma \zeta^{n+2} \|^{2}+\frac{77}{121} k \nu a\left(\zeta^{n+3}, \zeta^{n+3}\right) \\
&-\frac{43}{121} k \nu a\left(\zeta^{n+2}, \zeta^{n+2}\right)-\frac{13}{121} k \nu a\left(\zeta^{n+1}, \zeta^{n+1}\right) \\
& \leqslant c k\left(k^{3}+h^{r}\right)^{2}+k\left\{c^{\prime}\left(k^{3}+h^{r-1}\right)+\frac{24}{11} \frac{C_{b}}{C_{a}}\|u\|_{\left.L^{\infty}\left(\mathbf{H}^{1}\right)\right\}}\right. \\
& \cdot\left\{a\left(\Lambda \zeta^{n+3}, \Lambda \zeta^{n+3}\right) a\left(\Gamma \zeta^{n+3}, \Gamma \zeta^{n+3}\right)\right\}^{1 / 2}+C k\left\|\Lambda \zeta^{n+3}\right\|_{1}^{4}, \\
& 3 \leqslant n \leqslant J-3 .
\end{aligned}
$$

(In (2.40) $c^{\prime}$ is again of the form (2.39), albeit with different $C$.)

The triangle inequality and the arithmetic-geometric mean inequality yield, if we recall the definitions of $\Lambda \zeta^{n+3}, \Gamma \zeta^{n+3}$, the (convenient) estimate

$$
\begin{aligned}
& a\left(\Lambda \zeta^{n+3}, \Lambda \zeta^{n+3}\right)^{1 / 2} a\left(\Gamma \zeta^{n+3}, \Gamma \zeta^{n+3}\right)^{1 / 2} \\
& \quad \leqslant 5\left\{a\left(\zeta^{n+3}, \zeta^{n+3}\right)+a\left(\zeta^{n+2}, \zeta^{n+2}\right)+a\left(\zeta^{n+1}, \zeta^{n+1}\right)+a\left(\zeta^{n}, \zeta^{n}\right)\right\}
\end{aligned}
$$

We conclude then that if we take $k, h$ sufficiently small so that, e.g., the "cell Reynolds number"-type condition

$$
5 c^{\prime}\left(k^{3}+h^{r-1}\right) \leqslant \frac{2 \nu}{121}
$$

holds and in addition require, e.g., that

$$
\frac{120}{11} \frac{\dot{C}_{b}}{C_{a}}\|u\|_{L^{\infty}\left(\mathbf{H}^{1}\right)} \leqslant \frac{2 \nu}{121},
$$

i.e., a condition of the form (2.35), then (2.36) follows from (2.40)-(2.43).

Remark 2.1. As we remarked in the Introduction a condition of the form (2.35) on the solution of (1.1) is needed for the proof of uniqueness and regularity of the Navier-Stokes equations for $N=3$, cf. [18, p. 303], but is not necessary for $N=2$. It may be shown that it is guaranteed if $\nu$ is sufficiently large and/or the data $u^{0}, f$ are sufficiently small in appropriate function spaces; cf. Section 4 below. In Theorem 2.2 
we shall replace this condition by a requirement that $k$ and $h$ be sufficiently small; however this requirement will be qualitatively more stringent than, e.g., the one of the type (2.42).

We now complete by an inductive argument the estimation of $\left\|\zeta^{n+3}\right\|, 3 \leqslant n \leqslant$ $J-3$, starting from (2.36).

LEMMA 2.3. Let the hypotheses of Lemmata 2.1 and 2.2 be satisfied. In addition suppose that $h$ is further sufficiently small (so that (2.58) below is satisfied). Then, it follows that

$$
\left\|\zeta^{l}\right\|^{2}+k \nu a\left(\zeta^{l}, \zeta^{l}\right) \leqslant c(1+T)\left(k^{3}+h^{r}\right)^{2}, \quad 6 \leqslant l \leqslant J,
$$

where the constant $c$, as usual, does not depend on $T$ otherwise except through $L^{\alpha}(0, T ; X)$-norms of $u$ and $p$.

Proof. The proof proceeds by induction. For integer $M, 5 \leqslant M \leqslant J$, let $d_{M}=$ $d_{M}(u, p, k)$ be a constant (to be determined and bounded uniformly with respect to $M$ and $k$ later, cf. (2.56), (2.57)) such that

$$
\left\|\zeta^{l}\right\|+k^{1 / 2} \nu^{1 / 2}\left(a\left(\zeta^{l}, \zeta^{l}\right)\right)^{1 / 2} \leqslant d_{M-1}\left(k^{3}+h^{r}\right), \quad 3 \leqslant l \leqslant M-1,
$$

holds. Clearly (2.45) holds for $M=6$ with $d_{5}=\sqrt{2 c_{1}}$, with $c_{1}$ as in (2.27). We now have, using (1.3), (1.5), that

$$
\left\|\Lambda \zeta^{n+3}\right\|_{1}^{4} \leqslant C h^{-2}\left(\sum_{i=0}^{2}\left\|\zeta^{n+i}\right\|^{2}\right)\left[\sum_{i=0}^{2} a\left(\zeta^{n+i}, \zeta^{n+i}\right)\right] .
$$

Hence, using the induction hypothesis (2.45), we conclude that

$$
\left\|\Lambda \zeta^{n+3}\right\|_{1}^{4} \leqslant C_{1} d_{M-1}^{2} h^{-2}\left(k^{6}+h^{2 r}\right)\left[\sum_{i=0}^{2} a\left(\zeta^{n+i}, \zeta^{n+i}\right)\right], \quad n \leqslant M-3
$$

Now, using (2.26), we note that

$$
h^{-2}\left(k^{6}+h^{2 r}\right)=k^{6} h^{-2}+h^{2 r-2} \leqslant \alpha^{6} h^{10 / 7}+h^{2 r-2} .
$$

Hence, supposing that $h$ is sufficiently small, e.g., with $C$ as in (2.36) and $C_{1}$ as in (2.46), that

$$
C C_{1} d_{M-1}^{2}\left(\alpha^{6} h^{10 / 7}+h^{2 r-2}\right) \leqslant \nu / 121
$$

(note that eventually we shall replace $d_{M-1}$ in (2.47) by $\sup _{M, k}\left(d_{M}\right)-$ cf. (2.57) below), we obtain by $(2.36),(2.46),(2.47)$ that

$$
\begin{aligned}
\left\|\Gamma \zeta^{n+3}\right\|^{2}-\left\|\Gamma \zeta^{n+2}\right\|^{2} & +\frac{73}{121} k \nu a\left(\zeta^{n+3}, \zeta^{n+3}\right)-\frac{48}{121} k \nu a\left(\zeta^{n+2}, \zeta^{n+2}\right) \\
& -\frac{18}{121} k \nu a\left(\zeta^{n+1}, \zeta^{n+1}\right)-\frac{5 k \nu}{121} a\left(\zeta^{n}, \zeta^{n}\right) \\
\leqslant & c k\left(k^{3}+h^{r}\right)^{2}, \quad 3 \leqslant n \leqslant M-3
\end{aligned}
$$


Now we sum both sides of (2.48) with respect to $n$ from $n=3$ to $n=m-3$ where $m \leqslant M$, and we obtain

$$
\begin{aligned}
\left\|\Gamma \zeta^{m}\right\|^{2}- & \left\|\Gamma \zeta^{5}\right\|^{2}+\frac{73}{121} k \nu a\left(\zeta^{m}, \zeta^{m}\right) \\
\leqslant & \frac{k \nu}{121}\left[71 a\left(\zeta^{5}, \zeta^{5}\right)+23 a\left(\zeta^{4}, \zeta^{4}\right)+5 a\left(\zeta^{3}, \zeta^{3}\right)\right] \\
& +c k(m-5)\left(k^{3}+h^{r}\right)^{2}, \quad 6 \leqslant m \leqslant M
\end{aligned}
$$

Hence, using (2.27), we conclude that

$$
\left\|\Gamma \zeta^{m}\right\|+k^{1 / 2} \nu^{1 / 2}\left(a\left(\zeta^{m}, \zeta^{m}\right)\right)^{1 / 2} \leqslant E_{m-5}\left(k^{3}+h^{r}\right), \quad 6 \leqslant m \leqslant M,
$$

where

$$
E_{m}=E_{m}(u, p, k)=c \sqrt{1+k m} .
$$

(It is straightforward to show following the constants $c$ through the proof that the $c$ occurring in (2.50) is a positive polynomial function of $\left\|\partial^{4} u / \partial t^{4}\right\|_{L^{\infty}\left(L^{2}\right)}$, $\|\partial u / \partial t\|_{L^{\infty}\left(\mathbf{H}^{r}\right)}, \quad|\partial p / \partial t|_{L^{\infty}\left(H^{r-1} / R^{1}\right)}, \quad\|u\|_{L^{\infty}\left(\mathbf{W}^{1, \infty}\right)},\|u\|_{L^{\infty}\left(\mathbf{H}^{r}\right)}, \quad|p|_{L^{\infty}\left(H^{r-1} / R^{1}\right)}$, $\left\|\partial^{3} u / \partial t^{3}\right\|_{L^{\infty}\left(L^{\infty}\right)}$ and $c_{1}$ (as in (2.27)), with coefficients independent of $h, \hat{h}, k, m$ or T.) Recalling the definition of $\Gamma \zeta^{m}$, we may conclude from (2.49) that

$$
\begin{aligned}
\left\|\zeta^{m}\right\|-\frac{7}{11}\left\|\zeta^{m-1}\right\|-\frac{2}{11}\left\|\zeta^{m-2}\right\|+k^{1 / 2} \nu^{1 / 2}\left(a\left(\zeta^{m}, \zeta^{m}\right)\right)^{1 / 2} & \\
& \leqslant E_{m-5}\left(k^{3}+h^{r}\right), \quad 6 \leqslant m \leqslant M .
\end{aligned}
$$

Let $\lambda=(7+\sqrt{137}) / 22 \cong .85$ denote the root of the quadratic equation $x^{2}-7 x / 11$ $-2 / 11=0$ in the interval $(0,1)$. Multiply the $m$ th equation in $(2.51)$ by $\lambda^{6-m}$ and sum the resulting equations with respect to $m$ for $m=6$ to $m=M$ to obtain

$$
\begin{aligned}
& \sum_{m=6}^{M} \lambda^{6-m}\left(\left\|\zeta^{m}\right\|-\frac{7}{11}\left\|\zeta^{m-1}\right\|-\frac{2}{11}\left\|\zeta^{m-2}\right\|\right) \\
& \quad+k^{1 / 2} \nu^{1 / 2} \sum_{m=6}^{M} \lambda^{6-m}\left(a\left(\zeta^{m}, \zeta^{m}\right)\right)^{1 / 2} \leqslant\left(k^{3}+h^{r}\right) \sum_{m=6}^{M} \lambda^{6-m} E_{m-5} .
\end{aligned}
$$

Using the definition of $\lambda$, it is seen that

$$
\begin{aligned}
\sum_{m=6}^{M} \lambda^{6-m}\left(\left\|\zeta^{m}\right\|-\frac{7}{11}\left\|\zeta^{m-1}\right\|-\frac{2}{11}\left\|\zeta^{m-2}\right\|\right) & \\
= & \lambda^{6-M}\left\|\zeta^{M}\right\|+\frac{2}{11} \lambda^{5-M}\left\|\zeta^{M-1}\right\|-\left(\frac{7+2 \lambda^{-1}}{11}\right)\left\|\zeta^{5}\right\|-\frac{2}{11}\left\|\zeta^{4}\right\| \\
& \geqslant \lambda^{6-M}\left\|\zeta^{M}\right\|-\left(\frac{7+2 \lambda^{-1}}{11}\right)\left\|\zeta^{5}\right\|-\frac{2}{11}\left\|\zeta^{4}\right\|, \quad M \geqslant 6
\end{aligned}
$$

Hence from (2.52) we deduce that

$$
\begin{aligned}
& \left\|\zeta^{M}\right\|+k^{1 / 2} \nu^{1 / 2} \sum_{m=6}^{M} \lambda^{M-m}\left(a\left(\zeta^{m}, \zeta^{m}\right)\right)^{1 / 2} \\
& \quad \leqslant\left(\frac{7+2 \lambda^{-1}}{11}\right) \lambda^{M-6}\left\|\zeta^{5}\right\|+\frac{2}{11} \lambda^{M-6}\left\|\zeta^{4}\right\|+\left(k^{3}+h^{r}\right) \sum_{m=6}^{M} \lambda^{M-m} E_{m-5}
\end{aligned}
$$


Now, (2.50) gives

$$
E_{m-5}=c(1+k(m-5))^{1 / 2} \leqslant c(1+T)^{1 / 2} \text { if } 6 \leqslant m \leqslant J .
$$

Hence, since $0<\lambda<1, M \geqslant 6,(2.53)$ and (2.27) give

$$
\left\|\zeta^{M}\right\|+k^{1 / 2} \nu^{1 / 2}\left(a\left(\zeta^{M}, \zeta^{M}\right)\right)^{1 / 2} \leqslant d_{M}\left(k^{3}+h^{r}\right),
$$

where

$$
d_{M}=\left(\frac{7+2 \lambda^{-1}+2}{11}\right) c_{1}^{1 / 2}+\frac{c}{1-\lambda}(1+k(M-5))^{1 / 2} .
$$

In (2.56) $c_{1}, c$ are the constants occurring in (2.27), (2.50), respectively. By (2.56) we see that $d_{M}$ is an increasing function of $M$ and is bounded above uniformly in $k$ and $M$ by

$$
\sup _{k>0,6 \leqslant M \leqslant J} d_{M} \leqslant D=\left(\frac{7+2 \lambda^{-1}+2}{11}\right) c_{1}^{1 / 2}+\frac{c}{1-\lambda}(1+T)^{1 / 2} .
$$

Hence the induction proof started with (2.45) works if we define $d_{M}$ by (2.56) and we replace $d_{M-1}$ in (2.47) by the upper bound $D$ defined by (2.57). Hence, ultimately (2.47) is of the form

$$
c(1+T)\left(\alpha^{6} h^{10 / 7}+h^{2 r-2}\right) \leqslant \nu,
$$

which shows it to be qualitatively more stringent than, e.g., (2.42) which does not involve $T$ explicitly in its left-hand side. Finally then, if $h$ is sufficiently small so that (2.58) is satisfied, (2.45), (2.55), (2.57) give (2.44).

We now collect the results of the previous three lemmata in the main theorem of this section.

THEOREM 2.1. Let the initial conditions $U^{j}, j=0,1,2$, of (1.15) be chosen so that (2.25) is satisfied. Moreover, let (2.26) and (2.35) hold, and assume that $k$ and $h$ are sufficiently small, so that (2.42) and (2.58) are satisfied. Then, if $U^{n}$ and $u$ are solutions of (1.15), (1.1), respectively, we have that

$$
\max _{0 \leqslant n \leqslant J}\left\|U^{n}-u^{n}\right\| \leqslant c(1+T)^{1 / 2}\left(k^{3}+h^{r}\right) .
$$

Proof. (2.59) follows from (2.4), (2.25), (2.27), and (2.44).

The following error estimate for the pressure follows from Theorem 2.1.

Proposition 2.3. Let the hypotheses of Theorem 2.1 hold. Then, if $P^{n}, p$ are solutions of (1.15), (1.1), respectively, we have that

$$
\max _{3 \leqslant n \leqslant J}\left|P^{n}-p^{n}\right|_{L^{2} / R^{1}} \leqslant c(1+T)^{1 / 2}\left(k^{2}+k^{-1} h^{r}+h^{r-1}\right) .
$$

Proof. By (2.10) we see that

$$
\begin{aligned}
& B\left(\left[\zeta^{n+3}, e^{n+3}\right],[v, q]\right) \\
& =-\frac{11}{6 k}\left(\zeta^{n+3}-\frac{18}{11} \zeta^{n+2}+\frac{9}{11} \zeta^{n+1}-\frac{2}{11} \zeta^{n}, v\right) \\
& +\frac{11}{6 k}\left(\rho^{n+3}+\delta^{n+3}, v\right)+b\left(u^{n+3}, u^{n+3}, v\right)-b\left(\Lambda U^{n+3}, \Lambda U^{n+3}, v\right), \\
& \quad 0 \leqslant n \leqslant J-3, \forall[v, q] \in \mathbf{H}_{h, \hat{h} .}
\end{aligned}
$$


Hence, (2.2), (2.12)-(2.24) (noting that $\left(2.15^{\prime}\right),\left(2.21^{\prime}\right)$ hold for $n \geqslant 3$ as well), (2.30) and (2.31) yield

$$
\begin{aligned}
&\left|e^{n+3}\right|_{L^{2} / R^{1}} \leqslant C k^{-1}\left\|\zeta^{n+3}-\frac{18}{11} \zeta^{n+2}+\frac{9}{11} \zeta^{n+1}-\frac{2}{11} \zeta^{n}\right\| \\
&+c\left\{\left(k^{3}+h^{r}\right)+\left\|\Lambda \zeta^{n+3}\right\|+\left(k^{3}+h^{r-1}\right)\left\|\Lambda \zeta^{n+3}\right\|_{1}+\left\|\Lambda \zeta^{n+3}\right\|_{1}^{2}\right\} \\
& 0 \leqslant n \leqslant J-3 .
\end{aligned}
$$

Now, by (2.25), (2.27), (2.44),

$$
\begin{aligned}
k^{-1}\left\|\zeta^{n+3}-\frac{18}{11} \zeta^{n+2}+\frac{9}{11} \zeta^{n+1}-\frac{2}{11} \zeta^{n}\right\| \leqslant c\left(k^{2}+k^{-1} h^{r}\right), & \\
0 & \leqslant n \leqslant J-3 .
\end{aligned}
$$

Moreover, (1.5), (2.25), (2.27), (2.44), (2.26) and the facts that $k, h<1$ and $r \geqslant 2$ give

$$
\begin{aligned}
\left(k^{3}+h^{r-1}\right)\left\|\Lambda \zeta^{n+3}\right\|_{1} & \leqslant c\left(k^{3}+h^{r-1}\right)\left(k^{3} h^{-1}+h^{r-1}\right) \\
& =c\left(k^{6} h^{-1}+k^{3} h^{r-2}+k^{3} h^{r-1}+h^{2 r-2}\right) \\
& \leqslant c\left(k^{3}+h^{r}\right), \quad 0 \leqslant n \leqslant J-3,
\end{aligned}
$$

and

$$
\left\|\Lambda \zeta^{n+3}\right\|_{1}^{2} \leqslant c h^{-2}\left(k^{6}+h^{2 r}\right) \leqslant c\left(k^{2}+h^{r}\right), \quad 0 \leqslant n \leqslant J-3 .
$$

Hence, (2.61)-(2.64) and (2.25), (2.27), (2.44), and (2.5) yield (2.60).

Remark 2.2. (2.60) shows that, if we suppose, e.g., that $k \geqslant \alpha^{\prime} h$ for some positive constant $\alpha^{\prime}$ (a condition which for sufficiently small $h$ is certainly compatible with a condition of the form (2.26)), then $\left|P^{n}-p^{n}\right|_{L^{2} / R^{1}}=O\left(k^{2}+h^{r-1}\right)$.

We now state and prove a convergence result for which a condition of the type (2.35) is no longer needed. On the other hand a more stringent (than, e.g., the one given by (2.58)) "smallness" requirement on $k$ and $h$ will come into the picture.

THEOREM 2.2. Let the initial conditions $U^{j}, j=0,1,2$, of (1.15) be chosen so that (2.25) is satisfied. Moreover, let (2.26) hold, and assume that $k$ and $h$ are sufficiently small, so that (2.42) and (2.78) below are satisfied. Then if $\left[U^{n}, P^{n}\right]$ and $[u, p]$ are solutions of (1.15), (1.1), respectively, we have that

$$
\max _{0 \leqslant n \leqslant J}\left\|U^{n}-u^{n}\right\| \leqslant c e^{c T}\left(k^{3}+h^{r}\right)
$$

and

$$
\max _{3 \leqslant n \leqslant J}\left|P^{n}-p^{n}\right|_{L^{2} / R^{1}} \leqslant c e^{c T}\left(k^{2}+k^{-1} h^{r}+h^{r-1}\right)
$$

Proof. Obviously (2.27) still holds and so does (2.37). In (2.37) estimating the left-hand side as in Lemma 2.2 but estimating the right-hand side using (2.12)-(2.14), (2.16)-(2.20), (2.22)-(2.24) and (2.15') and $\left(2.21^{\prime}\right)$ (the last two obviously hold for $0 \leqslant n \leqslant J-3$ but they were used only for $n=0,1,2$ in the proof of Lemma 2.1), 
we obtain, instead of (2.38),

$$
\begin{aligned}
& \left\|\Gamma \zeta^{n+3}\right\|^{2}-\left\|\Gamma \zeta^{n+2}\right\|^{2}+\frac{78}{121} k \nu a\left(\zeta^{n+3}, \zeta^{n+3}\right) \\
& -\frac{42}{121} k \nu a\left(\zeta^{n+2}, \zeta^{n+2}\right)-\frac{12}{121} k \nu a\left(\zeta^{n+1}, \zeta^{n+1}\right) \\
& \leqslant 2\left\|\rho^{n+3}+\delta^{n+3}\right\|\left\|\Gamma \zeta^{n+3}\right\|_{1} \\
& +\frac{12}{11} k\left\{c\left(k^{3}+h^{r}\right)+c^{\prime}\left(k^{3}+h^{r-1}\right)\left\|\Lambda \zeta^{n+3}\right\|_{1}\right. \\
& \left.+c\left\|\Lambda \zeta^{n+3}\right\|+C_{b}\left\|\Lambda \zeta^{n+3}\right\|_{1}^{2}\right\}\left\|\Gamma \zeta^{n+3}\right\|_{1}, \\
& 3 \leqslant n \leqslant J-3 \text {, }
\end{aligned}
$$

with $c^{\prime}$ as in (2.39). Proceeding as in the proof of Lemma 2.2, we obtain

$$
\begin{aligned}
&\left\{2\left\|\rho^{n+3}+\delta^{n+3}\right\|\right.+\frac{12}{11} c k\left(k^{3}+h^{r}\right)+\frac{12 c k}{11}\left\|\Lambda \zeta^{n+3}\right\| \\
&\left.+\frac{12}{11} C_{b} k\left\|\Lambda \zeta^{n+3}\right\|_{1}^{2}\right\}\left\|\Gamma \zeta^{n+3}\right\|_{1} \\
& \leqslant c \varepsilon^{-1} k\left(k^{3}+h^{r}\right)^{2}+c \varepsilon^{-1} k\left\|\Lambda \zeta^{n+3}\right\|^{2}+C \varepsilon^{-1} k\left\|\Lambda \zeta^{n+3}\right\|_{1}^{4} \\
&+\varepsilon k\left[a\left(\zeta^{n+3}, \zeta^{n+3}\right)+a\left(\zeta^{n+2}, \zeta^{n+2}\right)+a\left(\zeta^{n+1}, \zeta^{n+1}\right)\right], \\
& 3 \leqslant n \leqslant J-3 .
\end{aligned}
$$

Choosing again $\varepsilon=\nu / 121$ gives, instead of (2.40),

$$
\begin{aligned}
&\left\|\Gamma \zeta^{n+3}\right\|^{2}-\left\|\Gamma \zeta^{n+2}\right\|^{2}+\frac{77}{121} k \nu a\left(\zeta^{n+3}, \zeta^{n+3}\right) \\
&-\frac{43}{121} k \nu a\left(\zeta^{n+2}, \zeta^{n+2}\right)-\frac{13}{121} k \nu a\left(\zeta^{n+1}, \zeta^{n+1}\right) \\
& \leqslant c k\left(k^{3}+h^{r}\right)^{2}+C k\left\|\Lambda \zeta^{n+3}\right\|_{1}^{4}+c^{\prime} k\left(k^{3}+h^{r-1}\right) \\
& \cdot\left\{a\left(\Lambda \zeta^{n+3}, \Lambda \zeta^{n+3}\right) a\left(\Gamma \zeta^{n+3}, \Gamma \zeta^{n+3}\right)\right\}^{1 / 2}+c k \sum_{i=0}^{2}\left\|\zeta^{n+i}\right\|^{2} \\
& 3 \leqslant n \leqslant J-3
\end{aligned}
$$

Applying (2.41) in the right-hand side of the above and choosing $k, h$ sufficiently small so that (2.42) is satisfied, we obtain, instead of (2.36),

$$
\begin{aligned}
& \left\|\Gamma \zeta^{n+3}\right\|^{2}-\left\|\Gamma \zeta^{n+2}\right\|^{2}+\frac{75}{121} k \nu a\left(\zeta^{n+3}, \zeta^{n+3}\right)-\frac{45}{121} k \nu a\left(\zeta^{n+2}, \zeta^{n+2}\right) \\
& \quad-\frac{15}{121} k \nu a\left(\zeta^{n+1}, \zeta^{n+1}\right)-\frac{2 k \nu}{121} a\left(\zeta^{n}, \zeta^{n}\right) \\
& \leqslant c k\left(k^{3}+h^{r}\right)^{2}+C k\left\|\Lambda \zeta^{n+3}\right\|_{1}^{4}+c k \sum_{i=0}^{2}\left\|\zeta^{n+i}\right\|^{2}, \quad 3 \leqslant n \leqslant J-3 .
\end{aligned}
$$

We now proceed as in the proof of Lemma 2.3. We assume again that

$$
\left\|\zeta^{l}\right\|+k^{1 / 2} \nu^{1 / 2} a\left(\zeta^{l}, \zeta^{l}\right) \leqslant \tilde{d}_{M-1}\left(k^{3}+h^{r}\right), \quad 3 \leqslant l \leqslant M-1,
$$

where $\tilde{d}_{M}=\tilde{d}_{M}(u, p, k)$ is a constant to be determined later. Assuming the analog of (2.47), i.e., that

$$
C C_{1} \tilde{d}_{M-1}^{2}\left(\alpha^{6} h^{10 / 7}+h^{2 r-2}\right) \leqslant \nu / 121
$$


we obtain, instead of (2.48),

$$
\begin{aligned}
& \left\|\Gamma \zeta^{n+3}\right\|^{2}-\left\|\Gamma \zeta^{n+2}\right\|^{2}+\frac{75}{121} k \nu a\left(\zeta^{n+3}, \zeta^{n+3}\right) \\
& -\frac{46}{121} k \nu a\left(\zeta^{n+2}, \zeta^{n+2}\right)-\frac{16}{121} k \nu a\left(\zeta^{n+1}, \zeta^{n+1}\right)-\frac{3 k \nu}{121} a\left(\zeta^{n}, \zeta^{n}\right) \\
& \quad \leqslant c k\left(k^{3}+h^{r}\right)^{2}+c k \sum_{i=0}^{2}\left\|\zeta^{n+i}\right\|^{2}, \quad 3 \leqslant n \leqslant M-3 .
\end{aligned}
$$

Summing both sides of the above with respect to $n$ from $n=3$ to $n=m-3$, $m \leqslant M$ gives

$$
\begin{aligned}
& \left\|\Gamma \zeta^{m}\right\|^{2}+\frac{75}{121} k \nu a\left(\zeta^{m}, \zeta^{m}\right) \\
& \leqslant C \sum_{j=3}^{5}\left[\left\|\zeta^{j}\right\|^{2}+k \nu a\left(\zeta^{j}, \zeta^{j}\right)\right]+c k(m-5)\left(k^{3}+h^{r}\right)^{2} \\
& \quad+c k \sum_{n=3}^{m-1}\left\|\zeta^{n}\right\|^{2}, \quad 6 \leqslant m \leqslant M
\end{aligned}
$$

from which it follows that the analog of $(2.49)$ is now

$$
\left\|\Gamma \zeta^{m}\right\|+k^{1 / 2} \nu^{1 / 2}\left(a\left(\zeta^{m}, \zeta^{m}\right)\right)^{1 / 2} \leqslant A_{m}^{1 / 2}, \quad 6 \leqslant m \leqslant M,
$$

where $\left\{A_{m}\right\}, m=6,7, \ldots, M$, is an increasing sequence of positive numbers, defined by

$$
A_{m}=c(1+k(m-5))\left(k^{3}+h^{r}\right)^{2}+c k \sum_{n=3}^{m-1}\left\|\zeta^{n}\right\|^{2} .
$$

Proceeding now as in the proof of Lemma 2.3 yields

$$
\begin{aligned}
\left\|\zeta^{M}\right\|+k^{1 / 2} \nu^{1 / 2} & \left(a\left(\zeta^{M}, \zeta^{M}\right)\right)^{1 / 2} \\
& \leqslant\left(\frac{7+2 \lambda^{-1}}{11}\right)\left\|\zeta^{5}\right\|+\frac{2}{11}\left\|\zeta^{4}\right\|+\frac{1}{1-\lambda} A_{M}^{1 / 2},
\end{aligned}
$$

from which it follows by (2.27) and (2.73) that

$$
\begin{aligned}
\left\|\zeta^{M}\right\|^{2} & +k \nu a\left(\zeta^{M}, \zeta^{M}\right) \\
& \leqslant \tilde{c}_{1}(1+T)\left(k^{3}+h^{r}\right)^{2}+\tilde{c}_{2} k \sum_{n=3}^{M-1}\left(\left\|\zeta^{n}\right\|^{2}+k \nu a\left(\zeta^{n}, \zeta^{n}\right)\right)
\end{aligned}
$$

with appropriate constants $\tilde{c}_{i}=\tilde{c}_{i}(u, p), i=1,2$. The discrete form of Gronwall's inequality gives then, by (2.74), that

$$
\left\|\zeta^{M}\right\|^{2}+k \nu a\left(\zeta^{M}, \zeta^{M}\right) \leqslant \tilde{c}_{1}(1+T)\left(k^{3}+h^{r}\right)^{2} \exp \left[\tilde{c}_{2} k(M-1)\right]
$$

i.e., that

$$
\left\|\zeta^{M}\right\|+k^{1 / 2} \nu^{1 / 2} a\left(\zeta^{M}, \zeta^{M}\right) \leqslant \tilde{d}_{M}\left(k^{3}+h^{r}\right)
$$

where

$$
\tilde{d}_{M}=\left\{2 \tilde{c}_{1}(1+T) \exp \left[\tilde{c}_{2} k(M-1)\right]\right\}^{1 / 2}
$$


Hence, $\tilde{d}_{M}$ is an increasing function of $M$ and is bounded above uniformly in $k$ and $M$ by

$$
\sup _{k>0,6 \leqslant M \leqslant J} \tilde{d}_{M} \leqslant \tilde{D}=\left\{2 \tilde{c}_{1}(1+T) \exp \left[\tilde{c}_{2} T\right]\right\}^{1 / 2} .
$$

Therefore, the induction proof started with (2.70) works if we define $\tilde{d}_{M}$ by $(2.75)$ and replace $\tilde{d}_{M-1}$ in (2.71) by $\tilde{D}$. Hence, ultimately, (2.71) is of the form

$$
c e^{c T}\left(\alpha^{6} h^{10 / 7}+h^{2 r-2}\right) \leqslant \nu
$$

which shows it to be qualitatively more stringent than (2.58). Hence, if $h$ is sufficiently small so that $(2.78)$ is satisfied, (2.70), (2.75), (2.76), (2.4), (2.25), (2.27) give (2.65). The estimate (2.66) for the pressure follows verbatim from Proposition 2.3 taking into account (2.65). Of course, Remark 2.2 is relevant here too.

3. Initial Conditions. In this section we consider the initial conditions $U^{j}, j=0,1,2$, constructed by (1.18)-(1.26), and we prove that they satisfy (2.25) for an appropriate constant $c_{0}=c_{0}(u, p)$. Clearly all the intermediate approximations needed for finding the initial conditions exist uniquely. The error estimates will be proved in a series of lemmata and will be summarized in Theorem 3.1.

LEMMA 3.1. Let $U^{0}$ be the solution of (1.18) and let $\omega^{0}=\omega(0)$ be defined by (2.3) for $t=0$. Then $U^{0} \in \tilde{\mathbf{S}}$ and

$$
\left\|U^{0}-\omega^{0}\right\| \leqslant c h^{r}
$$

and consequently

$$
\left\|U^{0}-u^{0}\right\| \leqslant c h^{r}
$$

Proof. That $U^{0} \in \tilde{\mathbf{S}}$ follows by putting $v=0$ in (1.18). We now define $\left[\bar{\omega}^{0}, \bar{\pi}^{0}\right] \in$ $\mathbf{H}_{h, \hat{h}}$ by

$$
B\left(\left[\bar{\omega}^{0}, \bar{\pi}^{0}\right],[v, q]\right)=B\left(\left[u^{0}, 0\right],[v, q]\right), \quad \forall[v, q] \in \mathbf{H}_{h, \hat{h}}
$$

We see that $\bar{\omega}^{0} \in \tilde{\mathbf{S}}$ and, putting

$$
\vec{\eta}^{0}=u^{0}-\bar{\omega}^{0}
$$

we see from (2.4) that

$$
\left\|\bar{\eta}^{0}\right\| \leqslant c h^{r}
$$

Let now

$$
\bar{\zeta}^{0}=U^{0}-\bar{\omega}^{0} .
$$

Then, (1.18) and (3.3) give that $\forall[v, q] \in \mathbf{H}_{h, \hat{h}}$

$$
\left(\bar{\zeta}^{0}, v\right)+\frac{6}{11} k B\left(\left[\bar{\zeta}^{0}, P^{0}-\bar{\pi}^{0}\right],[v, q]\right)=\left(\bar{\eta}^{0}, v\right) .
$$

Since by (3.6) $\bar{\zeta}^{0} \in \tilde{\mathbf{S}}$, putting $v=\bar{\zeta}^{0}$ in the above yields

$$
\left\|\bar{\zeta}^{0}\right\|^{2}+\frac{6}{11} k \nu a\left(\bar{\zeta}^{0}, \bar{\zeta}^{0}\right)=\left(\bar{\eta}^{0}, \bar{\zeta}^{0}\right) \leqslant \frac{1}{2}\left\|\bar{\eta}^{0}\right\|^{2}+\frac{1}{2}\left\|\bar{\zeta}^{0}\right\|^{2} .
$$

It follows by (3.5) that

$$
\left\|\bar{\zeta}^{0}\right\|^{2}+\frac{12}{11} k \nu a\left(\bar{\zeta}^{0}, \bar{\zeta}^{0}\right) \leqslant c h^{2 r}
$$


Since now $\left\|U^{0}-\omega^{0}\right\| \leqslant\left\|U^{0}-\bar{\omega}^{0}\right\|+\left\|u^{0}-\bar{\omega}^{0}\right\|+\left\|u^{0}-\omega^{0}\right\|$, it follows from (3.7), (3.5), (2.4) that (3.1) holds. By (2.4) we also obtain (3.2).

Lemma 3.2. Let $\tilde{U}^{6 / 11}$ be defined by (1.19), and let $\tilde{\zeta}^{6 / 11}=\tilde{U}^{6 / 11}-\omega^{6 / 11}$. Then $\tilde{U}^{6 / 11} \in \tilde{\mathbf{S}}$ and

$$
\left\|\tilde{\zeta}^{6 / 11}\right\|^{2}+k \nu a\left(\tilde{\zeta}^{6 / 11}, \tilde{\zeta}^{6 / 11}\right) \leqslant c\left(k^{2}+h^{r}\right)^{2}
$$

Proof. Putting $v=0$ in (1.19), we see that $\tilde{U}^{6 / 11} \in \tilde{\mathbf{S}}$. Letting $\tilde{\zeta}^{6 / 11}=\tilde{U}^{6 / 11}-$ $\omega^{6 / 11}, e^{6 / 11}=\tilde{P}^{6 / 11}-\pi^{6 / 11}$ (where of course, $\omega^{6 / 11}=\omega(6 k / 11), \pi^{6 / 11}=\pi(6 k / 11)$ are defined by (2.3)) and $\zeta^{0}=U^{0}-\omega^{0}$, we obtain, using (1.19), (2.3), that

$$
\begin{aligned}
\left(\tilde{\zeta}^{6 / 11}-\zeta^{0}, v\right)+ & \frac{6}{11} k B\left(\left[\tilde{\zeta}^{6 / 11}, \tilde{e}^{6 / 11}\right],[v, q]\right) \\
= & \left(\eta^{6 / 11}-\eta^{0}, v\right)-\left(u^{6 / 11}-u^{0}-\frac{6}{11} k u_{t}^{6 / 11}, v\right) \\
& +\frac{6 k}{11}\left[b\left(u^{6 / 11}, u^{6 / 11}, v\right)-b\left(u^{0}, u^{0}, v\right)\right], \quad \forall[v, q] \in \mathbf{H}_{h, \hat{h}} .
\end{aligned}
$$

Noting that $\tilde{\zeta}^{6 / 11}=\tilde{U}^{6 / 11}-\omega^{6 / 11} \in \tilde{\mathbf{S}}$, putting $v=\tilde{\zeta}^{6 / 11}$ in the above, and using the Cauchy-Schwarz inequality, we see that

$$
\begin{aligned}
\left\|\tilde{\zeta}^{6 / 11}\right\|^{2}+ & \frac{12}{11} k \nu a\left(\tilde{\zeta}^{6 / 11}, \tilde{\zeta}^{6 / 11}\right) \\
\leqslant & 2\left\|\eta^{6 / 11}-\eta^{0}\right\|\left\|\tilde{\zeta}^{6 / 11}\right\|+2\left\|u^{6 / 11}-u^{0}-\frac{6}{11} k u_{t}^{6 / 11}\right\|\left\|\tilde{\zeta}^{6 / 11}\right\| \\
& +\left\|\zeta^{0}\right\|^{2}+\frac{12 k}{11}\left|b\left(u^{6 / 11}, u^{6 / 11}, \tilde{\zeta}^{6 / 11}\right)-b\left(u^{0}, u^{0}, \tilde{\zeta}^{6 / 11}\right)\right|
\end{aligned}
$$

Now, (2.4) and Taylor's theorem with the remainder in integral form yield

$$
\begin{gathered}
\left\|\eta^{6 / 11}-\eta^{0}\right\| \leqslant \int_{0}^{6 k / 11}\left\|\eta_{t}(s)\right\| d s \leqslant c k h^{r} \\
\left\|u^{6 / 11}-u^{0}-\frac{6}{11} k u_{t}^{6 / 11}\right\| \leqslant c k^{2} .
\end{gathered}
$$

Moreover we have, by (2.9),

$$
\begin{aligned}
& k\left|b\left(u^{6 / 11}, u^{6 / 11}, \tilde{\zeta}^{6 / 11}\right)-b\left(u^{0}, u^{0}, \tilde{\zeta}^{6 / 11}\right)\right| \\
& \quad=k\left|b\left(u^{6 / 11}-u^{0}, u^{6 / 11}, \tilde{\zeta}^{6 / 11}\right)+b\left(u^{0}, u^{6 / 11}-u^{0}, \tilde{\zeta}^{6 / 11}\right)\right| \\
& \quad \leqslant k\left\|u^{6 / 11}-u^{0}\right\|\left\|u^{6 / 11}\right\|_{1, \infty}\left\|\tilde{\zeta}^{6 / 11}\right\|+k\left\|u^{0}\right\|\left\|u^{6 / 11}-u^{0}\right\|_{1, \infty}\left\|\tilde{\zeta}^{6 / 11}\right\| \\
& \quad \leqslant c k^{2}\left\|\tilde{\zeta}^{6 / 11}\right\| .
\end{aligned}
$$

Hence, (3.9)-(3.12), (3.1) and the arithmetic-geometric mean inequality yield (3.8).

Lemma 3.3. Let $\hat{U}_{1}$ be the solution of (1.20). Then $\hat{U}_{1} \in \tilde{\mathbf{S}}$. Assuming that

$$
k h^{-2 / 3} \leqslant \alpha,
$$

for any positive constant $\alpha$, then there exists a constant $c=c(u, p, \alpha)$ such that, if $\hat{\zeta}_{1}=\hat{U}_{1}-\frac{1}{2}\left(\omega^{12 / 11}+\omega^{0}\right)$, then

$$
\left\|\hat{\zeta}_{1}\right\|^{2}+k \nu a\left(\hat{\zeta}_{1}, \hat{\zeta}_{1}\right) \leqslant c\left(k^{5}+h^{2 r}\right)
$$


Proof. Clearly $\hat{U}_{1} \in \tilde{\mathbf{S}}$ as may be seen by putting $v=0$ in (1.20). We define $\hat{e}_{1}=\hat{P}_{1}-\frac{1}{2}\left(\pi^{12 / 11}+\pi^{0}\right)$ and $\zeta^{0}=U^{0}-\omega^{0}$ as usual. Then, (1.20), (2.3) yield

$$
\begin{aligned}
&\left(\hat{\zeta}_{1}-\zeta^{0}, v\right)+\frac{6}{11} k B\left(\left[\hat{\zeta}_{1}, \hat{e}_{1}\right],[v, q]\right) \\
&= \frac{1}{2}\left(\eta^{12 / 11}-\eta^{0}, v\right)-\frac{1}{2}\left(u^{12 / 11}-u^{0}-\frac{6 k}{11}\left(u_{t}^{12 / 11}+u_{t}^{0}\right), v\right) \\
&+ \frac{3 k}{11}\left[b\left(u^{12 / 11}, u^{12 / 11}, v\right)-b\left(2 \tilde{U}^{6 / 11}-u^{0}, 2 \tilde{U}^{6 / 11}-u^{0}, v\right)\right], \\
& \forall[v, q] \in \mathbf{H}_{h, \hat{h}}
\end{aligned}
$$

We have, using (2.4) and Taylor's theorem with remainder in integral form, that

$$
\begin{gathered}
\left\|\eta^{12 / 11}-\eta^{0}\right\| \leqslant c k h^{r} \\
\left\|u^{12 / 11}-u^{0}-\frac{6}{11} k\left(u_{t}^{12 / 11}+u_{t}^{0}\right)\right\| \leqslant c k^{3}
\end{gathered}
$$

We now have that

$$
\begin{aligned}
& b\left(u^{12 / 11}, u^{12 / 11}, v\right)-b\left(2 \tilde{U}^{6 / 11}-u^{0}, 2 \tilde{U}^{6 / 11}-u^{0}, v\right) \\
&= b\left(u^{12 / 11}-\left(2 \tilde{U}^{6 / 11}-u^{0}\right), u^{12 / 11}, v\right) \\
&+b\left(2 \tilde{U}^{6 / 11}-u^{0}, u^{12 / 11}-\left(2 \tilde{U}^{6 / 11}-u^{0}\right), v\right) \\
&= b\left(u^{12 / 11}-\tilde{\Lambda} u^{12 / 11}-2 \tilde{\zeta}^{6 / 11}+2 \eta^{6 / 11}, u^{12 / 11}, v\right) \\
&+b\left(2 \tilde{\zeta}^{6 / 11}-2 \eta^{6 / 11}, u^{12 / 11}-\tilde{\Lambda} u^{12 / 11}-2 \tilde{\zeta}^{6 / 11}+2 \eta^{6 / 11}, v\right) \\
&+b\left(\tilde{\Lambda} u^{12 / 11}, u^{12 / 11}-\tilde{\Lambda} u^{12 / 11}-2 \tilde{\zeta}^{6 / 11}+2 \eta^{6 / 11}, v\right)
\end{aligned}
$$

where $\tilde{\zeta}^{6 / 11}$ was defined in Lemma 3.2 and

$$
\tilde{\Lambda} u^{12 / 11} \equiv 2 u^{6 / 11}-u^{0} \text {. }
$$

We now estimate the three terms in the right-hand side of (3.18). For every $v \in \mathbf{S}_{h}^{r}$ we have first by (2.7) that

$$
\begin{aligned}
& k\left|b\left(u^{12 / 11}-\tilde{\Lambda} u^{12 / 11}-2 \tilde{\zeta}^{6 / 11}+2 \eta^{6 / 11}, u^{12 / 11}, v\right)\right| \\
& \quad \leqslant C k\left\|u^{12 / 11}-\tilde{\Lambda} u^{12 / 11}-2 \tilde{\zeta}^{6 / 11}+2 \eta^{6 / 11}\right\|\left\|u^{12 / 11}\right\|_{1, \infty}\|v\|_{1}
\end{aligned}
$$

Now (3.19) gives

$$
\left\|u^{12 / 11}-\tilde{\Lambda} u^{12 / 11}\right\|_{j} \leqslant c k^{2}, \quad j=0,1
$$

We conclude by (3.20), the triangle inequality, (3.21), (3.8), (2.4), (1.3) and from the weighted arithmetic-geometric mean inequality that, for any $\varepsilon>0$,

$$
\begin{aligned}
k \mid b\left(u^{12 / 11}-\tilde{\Lambda} u^{12 / 11}-\right. & \left.2 \tilde{\xi}^{6 / 11}+2 \eta^{6 / 11}, u^{12 / 11}, v\right) \mid \\
& \leqslant c \varepsilon^{-1} k\left(k^{2}+h^{r}\right)^{2}+\varepsilon k a(v, v) \\
& \leqslant c \varepsilon^{-1}\left(k^{5}+h^{2 r}\right)+\varepsilon k a(v, v), \quad \forall v \in \mathbf{S}_{h}^{r}
\end{aligned}
$$


For the second term in the right-hand side of (3.18) we obtain, using (1.11) and (1.3), for any $\varepsilon>0, v \in \mathbf{S}_{h}^{r}$

$$
\begin{aligned}
& k\left|b\left(2 \tilde{\zeta}^{6 / 11}-2 \eta^{6 / 11}, u^{12 / 11}-\tilde{\Lambda} u^{12 / 11}-2 \tilde{\zeta}^{6 / 11}+2 \eta^{6 / 11}, v\right)\right| \\
& \leqslant C_{b} k\left\|2 \tilde{\zeta}^{6 / 11}-2 \eta^{6 / 11}\right\|_{1}\left\|u^{12 / 11}-\tilde{\Lambda} u^{12 / 11}\right\|_{1}\|v\|_{1} \\
&+C_{b} k\left\|2 \tilde{\zeta}^{6 / 11}-2 \eta^{6 / 11}\right\|_{1}^{2}\|v\|_{1} \\
& \leqslant C k \varepsilon^{-1}\left(\left\|\tilde{\zeta}^{6 / 11}-\eta^{6 / 11}\right\|_{1}^{2}\left\|u^{12 / 11}-\tilde{\Lambda} u^{12 / 11}\right\|_{1}^{2}+\left\|\tilde{\zeta}^{6 / 11}-\eta^{6 / 11}\right\|_{1}^{4}\right) \\
&+k \varepsilon a(v, v) .
\end{aligned}
$$

Now, using (3.21) yields

$$
\begin{aligned}
k\left\|\tilde{\zeta}^{6 / 11}-\eta^{6 / 11}\right\|_{1}^{2}\left\|u^{12 / 11}-\tilde{\Lambda} u^{12 / 11}\right\|_{1}^{2}+k\left\|\tilde{\zeta}^{6 / 11}-\eta^{6 / 11}\right\|_{1}^{4} \\
\quad \leqslant c k^{5}\left(\left\|\tilde{\zeta}^{6 / 11}\right\|_{1}^{2}+\left\|\eta^{6 / 11}\right\|_{1}^{2}\right)+C k\left(\left\|\tilde{\zeta}^{6 / 11}\right\|_{1}^{4}+\left\|\eta^{6 / 11}\right\|_{1}^{4}\right) .
\end{aligned}
$$

Now, (3.8), (2.4) and the facts that $r \geqslant 2, k, h<1$, yield

$$
\begin{aligned}
k^{5}\left(\left\|\tilde{\zeta}^{6 / 11}\right\|_{1}^{2}+\left\|\eta^{6 / 11}\right\|_{1}^{2}\right) & \leqslant C k^{4}\left(\nu k a\left(\tilde{\zeta}^{6 / 11}, \tilde{\zeta}^{6 / 11}\right)\right)+c k^{5} h^{2 r-2} \\
& \leqslant c\left(k^{8}+k^{4} h^{2 r}+k^{5} h^{2 r-2}\right) \leqslant c\left(k^{5}+h^{2 r}\right)
\end{aligned}
$$

and

$$
k\left\|\eta^{6 / 11}\right\|_{1}^{4} \leqslant c k h^{4 r-4} \leqslant c h^{2 r} .
$$

Also, (3.8), (1.5), (3.13) and the facts that $r \geqslant 2, k, h<1$ yield

$$
\begin{aligned}
k\left\|\tilde{\zeta}^{6 / 11}\right\|_{1}^{4} & \leqslant C k \nu a\left(\tilde{\zeta}^{6 / 11}, \tilde{\zeta}^{6 / 11}\right)\left\|\tilde{\zeta}^{6 / 11}\right\|_{1}^{2} \\
& \leqslant c\left(k^{4}+h^{2 r}\right) h^{-2}\left(k^{4}+h^{2 r}\right) \leqslant c\left(k^{8} h^{-2}+k^{4} h^{2 r-2}+h^{4 r-2}\right) \\
& \leqslant c\left(\alpha^{3} k^{5}+\alpha^{3} k h^{2 r}+h^{4 r-2}\right) \leqslant c\left(k^{5}+h^{2 r}\right) .
\end{aligned}
$$

Hence, (3.23)-(3.27) yield for any $\varepsilon>0, v \in \mathbf{S}_{h}^{r}$

$$
\begin{aligned}
k \mid b\left(2 \tilde{\zeta}^{6 / 11}-2 \eta^{6 / 11}, u^{12 / 11}-\tilde{\Lambda} u^{12 / 11}-\right. & \left.2 \tilde{\zeta}^{6 / 11}+2 \eta^{6 / 11}, v\right) \mid \\
& \leqslant c \varepsilon^{-1}\left(k^{5}+h^{2 r}\right)+k \varepsilon a(v, v) .
\end{aligned}
$$

Finally, for the third term in the right-hand side of (3.18) we obtain, using (1.3), (2.8), (3.19), (3.21), (3.8), (2.4) for any $\varepsilon>0, v \in \mathbf{S}_{h}^{r}$

$$
\begin{aligned}
& k\left|b\left(\tilde{\Lambda} u^{12 / 11}, u^{12 / 11}-\tilde{\Lambda} u^{12 / 11}-2 \tilde{\zeta}^{6 / 11}+2 \eta^{6 / 11}, v\right)\right| \\
& \leqslant C k\left\|\tilde{\Lambda} u^{12 / 11}\right\|_{\infty}\left(\left\|u^{12 / 11}-\tilde{\Lambda} u^{12 / 11}\right\|+\left\|\tilde{\zeta}^{6 / 11}\right\|+\left\|\eta^{6 / 11}\right\|\right)\|v\|_{1} \\
& \leqslant c k\left(k^{2}+h^{r}\right)\|v\|_{1} \leqslant c \varepsilon^{-1}\left(k^{5}+h^{2 r}\right)+k \varepsilon a(v, v) .
\end{aligned}
$$

Now, putting $v=\hat{\zeta}_{1} \in \tilde{\mathbf{S}}$ in (3.15), we obtain, using the standard inequalities, (3.16), (3.17), (3.22), (3.28) and the above, for any $\varepsilon, \varepsilon^{\prime}>0$,

$$
\begin{aligned}
\frac{1}{2}\left\|\hat{\zeta}_{1}\right\|^{2}+\frac{6}{11} k \nu a\left(\hat{\zeta}_{1}, \hat{\zeta}_{1}\right) & \\
\leqslant & \frac{1}{2}\left\|\zeta^{0}\right\|^{2}+c\left(\varepsilon^{\prime}\right)^{-1}\left(k h^{r}\right)^{2}+\varepsilon^{\prime}\left\|\hat{\zeta}_{1}\right\|^{2} \\
& +c\left(\varepsilon^{\prime}\right)^{-1} k^{6}+\varepsilon^{\prime}\left\|\hat{\zeta}_{1}\right\|^{2}+c \varepsilon^{-1}\left(k^{5}+h^{2 r}\right)+3 \varepsilon k a\left(\hat{\zeta}_{1}, \hat{\zeta}_{1}\right),
\end{aligned}
$$

which, with the choice of, for example, $\varepsilon^{\prime}=1 / 8, \varepsilon=\nu / 33$ and (3.1) yields (3.14). 
Lemma 3.4. Let $\hat{U}_{2}$ be the solution of (1.21). Then $\hat{U}_{2} \in \tilde{\mathbf{S}}$. Moreover, if (3.13) holds and $\hat{\zeta}_{2}=\hat{U}_{2}-\frac{1}{2}\left(\omega^{12 / 11}+\omega^{0}\right)$, we have that

$$
\left\|\hat{\zeta}_{2}\right\|^{2}+k \nu a\left(\hat{\zeta}_{2}, \hat{\zeta}_{2}\right) \leqslant c\left(k^{3}+h^{r}\right)^{2} \text {. }
$$

Proof. Clearly $\hat{U}_{2} \in \tilde{\mathbf{S}}$ (put $v=0$ in (1.21)). Define now $\hat{e}_{2}=\hat{P}_{2}-\frac{1}{2}\left(\pi^{12 / 11}+\pi^{0}\right)$ and $\zeta^{0}=U^{0}-\omega^{0}$ as usual. Then, (1.21), (2.3) yield

$$
\begin{aligned}
&\left(\hat{\zeta}_{2}-\zeta^{0}, v\right)+ \frac{6}{11} k B\left(\left[\hat{\zeta}_{2}, \hat{e}_{2}\right],[v, q]\right) \\
&= \frac{1}{2}\left(\eta^{12 / 11}-\eta^{0}, v\right)-\frac{1}{2}\left(u^{12 / 11}-u^{0}-\frac{6 k}{11}\left(u_{t}^{0}+u_{t}^{12 / 11}\right), v\right) \\
&+\frac{3 k}{11}\left\{b\left(u^{12 / 11}, u^{12 / 11}, v\right)-b\left(2 \hat{U}_{1}-u^{0}, 2 \hat{U}_{1}-u^{0}, v\right)\right\}, \\
& \forall[v, q] \in \mathbf{H}_{h, \hat{h}}
\end{aligned}
$$

Now we see that

$$
\begin{aligned}
b\left(u^{12 / 11},\right. & \left.u^{12 / 11}, v\right)-b\left(2 \hat{U}_{1}-u^{0}, 2 \hat{U}_{1}-u^{0}, v\right) \\
= & b\left(u^{12 / 11}-\left(2 \hat{U}_{1}-u^{0}\right), u^{12 / 11}, v\right) \\
& +b\left(2 \hat{U}_{1}-u^{0}, u^{12 / 11}-\left(2 \hat{U}_{1}-u^{0}\right), v\right) \\
= & b\left(\eta^{12 / 11}-2 \hat{\zeta}_{1}+\eta^{0}, u^{12 / 11}, v\right) \\
& +b\left(u^{12 / 11}-\eta^{12 / 11}+2 \hat{\zeta}_{1}-\eta^{0}, \eta^{12 / 11}-2 \hat{\zeta}_{1}+\eta^{0}, v\right) \\
= & b\left(\eta^{12 / 11}-2 \hat{\zeta}_{1}+\eta^{0}, u^{12 / 11}, v\right)+b\left(u^{12 / 11}, \eta^{12 / 11}-2 \hat{\zeta}_{1}+\eta^{0}, v\right) \\
& -b\left(\eta^{12 / 11}-2 \hat{\zeta}_{1}+\eta^{0}, \eta^{12 / 11}-2 \hat{\zeta}_{1}+\eta^{0}, v\right),
\end{aligned}
$$

where $\hat{\zeta}_{1}$ was defined in Lemma 3.3.

For the first term in the right-hand side of (3.31) we obtain using (2.7), (2.4), (3.14), for any $\varepsilon>0, v \in \mathbf{S}_{h}^{r}$ that

$$
\begin{aligned}
k & \left|b\left(\eta^{12 / 11}-2 \hat{\zeta}_{1}+\eta^{0}, u^{12 / 11}, v\right)\right| \\
& \leqslant C k\left\|\eta^{12 / 11}-2 \hat{\zeta}_{1}+\eta^{0}\right\|\left\|u^{12 / 11}\right\|_{1, \infty}\|v\|_{1} \\
& \leqslant c k\left(\left\|\eta^{12 / 11}\right\|+\left\|\hat{\zeta}_{1}\right\|+\left\|\eta^{0}\right\|\right)\|v\|_{1} \leqslant c k\left(k^{5 / 2}+h^{r}\right)\|v\|_{1} \\
& \leqslant c \varepsilon^{-1} k\left(k^{5}+h^{2 r}\right)+C_{a} \varepsilon k\|v\|_{1}^{2} \leqslant c \varepsilon^{-1}\left(k^{6}+h^{2 r}\right)+\varepsilon k a(v, v) .
\end{aligned}
$$

Similarly, for the second term, using (2.8) and then exactly as above, for any $v \in \mathbf{S}_{h}^{r}$, $\varepsilon>0$,

$$
\begin{aligned}
k\left|b\left(u^{12 / 11}, \eta^{12 / 11}-2 \hat{\zeta}_{1}+\eta^{0}, v\right)\right| \\
\quad \leqslant C k\left\|u^{12 / 11}\right\|_{\infty}\left\|\eta^{12 / 11}-2 \hat{\zeta}_{1}+\eta^{0}\right\|\|v\|_{1} \\
\quad \leqslant c \varepsilon^{-1}\left(k^{6}+h^{2 r}\right)+\varepsilon k a(v, v) .
\end{aligned}
$$

We estimate now the third term of the right-hand side of (3.31). Using (1.11) we see that, for $\varepsilon>0$,

$$
\begin{aligned}
k\left|b\left(\eta^{12 / 11}-2 \hat{\zeta}_{1}+\eta^{0}, \eta^{12 / 11}-2 \hat{\zeta}_{1}+\eta^{0}, v\right)\right| \\
\quad \leqslant k C_{b}\left\|\eta^{12 / 11}-2 \hat{\zeta}_{1}+\eta^{0}\right\|_{1}^{2}\|v\|_{1} \\
\quad \leqslant C \varepsilon^{-1} k\left\|\eta^{12 / 11}-2 \hat{\zeta}_{1}+\eta^{0}\right\|_{1}^{4}+\varepsilon k a(v, v) .
\end{aligned}
$$


Now, (2.4), (3.14), (1.5), (3.13), and the facts that $k, h<1, r \geqslant 2$ give

$$
\begin{aligned}
k \| \eta^{12 / 11} & -2 \hat{\zeta}_{1}+\eta^{0}\left\|_{1}^{4} \leqslant C k\left(\left\|\eta^{12 / 11}\right\|_{1}^{4}+\left\|\eta^{0}\right\|_{1}^{4}\right)+C k\right\| \hat{\zeta}_{1} \|_{1}^{4} \\
& \leqslant c k h^{4 r-4}+C\left(k \nu a\left(\hat{\zeta}_{1}, \hat{\zeta}_{1}\right)\right)\left\|\hat{\zeta}_{1}\right\|_{1}^{2} \leqslant c k h^{4 r-4}+c\left(k^{5}+h^{2 r}\right) h^{-2}\left(k^{5}+h^{2 r}\right) \\
& \leqslant c\left(k h^{4 r-4}+k^{10} h^{-2}+k^{5} h^{2 r-2}+h^{4 r-2}\right) \\
& \leqslant c\left(h^{2 r}+\alpha^{4} k^{6} h^{2 / 3}+\alpha^{5} h^{2 r+4 / 3}+h^{4 r-2}\right) \leqslant c\left(k^{6}+h^{2 r}\right) .
\end{aligned}
$$

Hence (3.34) becomes, $\forall \varepsilon>0, v \in \mathbf{S}_{h}^{r}$ :

$$
\begin{gathered}
k\left|b\left(\eta^{12 / 11}-2 \hat{\zeta}_{1}+\eta^{0}, \eta^{12 / 11}-2 \hat{\zeta}_{1}+\eta^{0}, v\right)\right| \\
\leqslant c \varepsilon^{-1}\left(k^{6}+h^{2 r}\right)+\varepsilon k a(v, v) .
\end{gathered}
$$

Picking now $v=\hat{\zeta}_{2} \in \tilde{\mathbf{S}}$ in (3.30) and proceeding as in the previous lemma, we see that (3.31)-(3.35), (3.16), (3.17) and (3.1) yield (3.29).

We now let $U^{12 / 11}$ be defined by (1.22). Clearly $U^{12 / 11} \in \tilde{\mathbf{S}}$. Defining $\zeta^{12 / 11}$ by

$$
\zeta^{12 / 11}=U^{12 / 11}-\omega^{12 / 11},
$$

we see by (1.22), the definition of $\hat{\zeta}_{2},(3.29)$ and (3.1) that

$$
\begin{aligned}
\left\|\zeta^{12 / 11}\right\| & =\left\|2 \hat{U}_{2}-U^{0}-\omega^{12 / 11}\right\|=\left\|2 \hat{\zeta}_{2}+\omega^{0}-U^{0}\right\| \\
& \leqslant 2\left\|\hat{\zeta}_{2}\right\|+\left\|\omega^{0}-U^{0}\right\| \leqslant c\left(k^{3}+h^{r}\right) .
\end{aligned}
$$

Lemma 3.5. Let $\hat{U}_{3}$ be the solution of (1.23). Then $\hat{U}_{3} \in \tilde{\mathbf{S}}$. Moreover, if (3.13) holds and $\hat{\zeta}_{3}=\hat{U}_{3}-\frac{1}{2}\left(\omega^{24 / 11}+\omega^{12 / 11}\right)$, we have

$$
\left\|\hat{\zeta}_{3}\right\|^{2}+k \nu a\left(\hat{\zeta}_{3}, \hat{\zeta}_{3}\right) \leqslant c\left(k^{3}+h^{r}\right)^{2}
$$

Proof. Putting $v=0$ in (1.23), we see that $\hat{U}_{3} \in \tilde{\mathbf{S}}$. We define now $\hat{e}_{3}=\hat{P}_{3}$ $-\frac{1}{2}\left(\pi^{24 / 11}+\pi^{12 / 11}\right)$ and $\zeta^{12 / 11}$ by (3.36). (Note for future reference that $\hat{\zeta}_{3} \in \tilde{\mathbf{S}}$.) Using (1.23) and (2.3), we obtain that

$$
\begin{aligned}
\left(\hat{\zeta}_{3}-\zeta^{12 / 11}, v\right)+\frac{6 k}{11} B\left(\left[\hat{\zeta}_{3}, \hat{e}_{3}\right],[v, q]\right) & \\
= & \frac{1}{2}\left(\eta^{24 / 11}-\eta^{12 / 11}, v\right) \\
& -\frac{1}{2}\left(u^{24 / 11}-u^{12 / 11}-\frac{6 k}{11}\left(u_{t}^{24 / 11}+u_{t}^{12 / 11}\right), v\right) \\
& +\frac{3 k}{11}\left[b\left(u^{12 / 11}, u^{12 / 11}, v\right)-b\left(U^{12 / 11}, U^{12 / 11}, v\right)\right] \\
& +\frac{3 k}{11}\left[b\left(u^{24 / 11}, u^{24 / 11}, v\right)-b\left(2 U^{12 / 11}-u^{0}, 2 U^{12 / 11}-u^{0}, v\right)\right], \\
& \forall[v, q] \in \mathbf{H}_{h, \hat{h}} .
\end{aligned}
$$

Now we write the third term of the right-hand side of the above as

$$
\begin{aligned}
& b\left(u^{12 / 11}, u^{12 / 11}, v\right)-b\left(U^{12 / 11}, U^{12 / 11}, v\right) \\
&= b\left(u^{12 / 11}-U^{12 / 11}, u^{12 / 11}, v\right)+b\left(U^{12 / 11}, u^{12 / 11}-U^{12 / 11}, v\right) \\
&= b\left(\eta^{12 / 11}-\zeta^{12 / 11}, u^{12 / 11}, v\right)+b\left(u^{12 / 11}, \eta^{12 / 11}-\zeta^{12 / 11}, v\right) \\
&-b\left(\eta^{12 / 11}-\zeta^{12 / 11}, \eta^{12 / 11}-\zeta^{12 / 11}, v\right), \quad \forall v \in \mathbf{S}_{h}^{r} .
\end{aligned}
$$


From (2.7), (2.4) and (3.37) we see for $\varepsilon>0, v \in \mathbf{S}_{h}^{r}$ that

$$
\begin{aligned}
& k\left|b\left(\eta^{12 / 11}-\zeta^{12 / 11}, u^{12 / 11}, v\right)\right| \\
& \quad \leqslant C k\left\|\eta^{12 / 11}-\zeta^{12 / 11}\right\|\left\|u^{12 / 11}\right\|_{1, \infty}\|v\|_{1} \\
& \quad \leqslant c k\left(\left\|\eta^{12 / 11}\right\|+\left\|\zeta^{12 / 11}\right\|\right)\|v\|_{1} \leqslant c \varepsilon^{-1} k\left(k^{6}+h^{2 r}\right)+\varepsilon k a(v, v) .
\end{aligned}
$$

From (2.8), (2.4) and (3.37), for $\varepsilon>0, v \in \mathbf{S}_{h}^{r}$

$$
\begin{aligned}
k\left|b\left(u^{12 / 11}, \eta^{12 / 11}-\zeta^{12 / 11}, v\right)\right| & \\
& \leqslant C k\left\|u^{12 / 11}\right\|_{\infty}\left\|\eta^{12 / 11}-\zeta^{12 / 11}\right\|\|v\|_{1} \\
& \leqslant c \varepsilon^{-1} k\left(k^{6}+h^{2 r}\right)+\varepsilon k a(v, v) .
\end{aligned}
$$

Finally, using (1.11), (2.4), (1.5), (3.37), (3.13), $r \geqslant 2$ yields for $\varepsilon>0, v \in \mathbf{S}_{h}^{r}$

$$
\begin{aligned}
k & \left|b\left(\eta^{12 / 11}-\zeta^{12 / 11}, \eta^{12 / 11}-\zeta^{12 / 11}, v\right)\right| \\
& \leqslant C_{b} k\left\|\eta^{12 / 11}-\zeta^{12 / 11}\right\|_{1}^{2}\|v\|_{1} \leqslant C k\left(\left\|\eta^{12 / 11}\right\|_{1}^{2}+\left\|\zeta^{12 / 11}\right\|_{1}^{2}\right)\|v\|_{1} \\
& \leqslant c k\left(h^{2 r-2}+h^{-2} k^{6}+h^{2 r-2}\right)\|v\|_{1} \leqslant c \varepsilon^{-1} k\left(k^{12} h^{-4}+h^{4 r-4}\right)+\varepsilon k a(v, v) \\
& \leqslant c \varepsilon^{-1} k\left(\alpha^{6} k^{7}+h^{2 r}\right)+\varepsilon k a(v, v) \leqslant c \varepsilon^{-1} k\left(k^{6}+h^{2 r}\right)+\varepsilon k a(v, v) .
\end{aligned}
$$

Hence, (3.40)-(3.43) give for $\varepsilon>0, v \in \mathbf{S}_{h}^{r}$,

$$
\begin{gathered}
k\left|b\left(u^{12 / 11}, u^{12 / 11}, v\right)-b\left(U^{12 / 11}, U^{12 / 11}, v\right)\right| \\
\leqslant c \varepsilon^{-1} k\left(k^{6}+h^{2 r}\right)+\varepsilon k a(v, v) .
\end{gathered}
$$

To estimate now the fourth term of the right-hand side of (3.39) we define $\tilde{\Lambda} u^{24 / 11}=2 u^{12 / 11}-u^{0}, \tilde{\Lambda} U^{24 / 11}=2 U^{12 / 11}-u^{0}, \tilde{\Lambda} \omega^{24 / 11}=2 \omega^{12 / 11}-\omega^{0}$ and obtain

$$
\text { (3.45) } \begin{aligned}
b & \left(u^{24 / 11}, u^{24 / 11}, v\right)-b\left(2 U^{12 / 11}-u^{0}, 2 U^{12 / 11}-u^{0}, v\right) \\
= & b\left(\tilde{\Lambda} u^{24 / 11}, u^{24 / 11}-\tilde{\Lambda} u^{24 / 11}, v\right)+b\left(\tilde{\Lambda} u^{24 / 11}, \tilde{\Lambda} u^{24 / 11}-\tilde{\Lambda} \omega^{24 / 11}, v\right) \\
& +b\left(\tilde{\Lambda} u^{24 / 11}, \tilde{\Lambda} \omega^{24 / 11}-\tilde{\Lambda} U^{24 / 11}, v\right)+b\left(u^{24 / 11}-\tilde{\Lambda} u^{24 / 11}, u^{24 / 11}, v\right) \\
& +b\left(\tilde{\Lambda} u^{24 / 11}-\tilde{\Lambda} \omega^{24 / 11}, \tilde{\Lambda} u^{24 / 11}, v\right)+b\left(\tilde{\Lambda} \omega^{24 / 11}-\tilde{\Lambda} U^{24 / 11}, \tilde{\Lambda} u^{24 / 11}, v\right) \\
& -b\left(\tilde{\Lambda} u^{24 / 11}-\tilde{\Lambda} \omega^{24 / 11}, \tilde{\Lambda} u^{24 / 11}-\tilde{\Lambda} \omega^{24 / 11}, v\right) \\
& -b\left(\tilde{\Lambda} u^{24 / 11}-\tilde{\Lambda} \omega^{24 / 11}, \tilde{\Lambda} \omega^{24 / 11}-\tilde{\Lambda} U^{24 / 11}, v\right) \\
& -b\left(\tilde{\Lambda} \omega^{24 / 11}-\tilde{\Lambda} U^{24 / 11}, \tilde{\Lambda} u^{24 / 11}-\tilde{\Lambda} \omega^{24 / 11}, v\right) \\
& -b\left(\tilde{\Lambda} \omega^{24 / 11}-\tilde{\Lambda} U^{24 / 11}, \tilde{\Lambda} \omega^{24 / 11}-\tilde{\Lambda} U^{24 / 11}, v\right)
\end{aligned}
$$

By Taylor's theorem with remainder in integral form we obtain

$$
\left\|u^{24 / 11}-\tilde{\Lambda} u^{24 / 11}\right\|_{j} \leqslant c k^{2}, \quad j=0,1 .
$$

From (2.8'), (3.46), for $\varepsilon^{\prime}>0$,

$$
\begin{aligned}
& k\left|b\left(\tilde{\Lambda} u^{24 / 11}, u^{24 / 11}-\tilde{\Lambda} u^{24 / 11}, v\right)\right| \\
& \quad \leqslant C k\left\|\tilde{\Lambda} u^{24 / 11}\right\|_{\infty}\left\|u^{24 / 11}-\tilde{\Lambda} u^{24 / 11}\right\|_{1}\|v\| \\
& \quad \leqslant c k^{3}\|v\| \leqslant c\left(\varepsilon^{\prime}\right)^{-1} k^{6}+\varepsilon^{\prime}\|v\|^{2} .
\end{aligned}
$$


From (2.8), (2.4), for $\varepsilon>0$,

$$
\begin{aligned}
& k\left|b\left(\tilde{\Lambda} u^{24 / 11}, \tilde{\Lambda} u^{24 / 11}-\tilde{\Lambda} \omega^{24 / 11}, v\right)\right| \\
& \quad \leqslant C k\left\|\tilde{\Lambda} u^{24 / 11}\right\|_{\infty}\left\|\tilde{\Lambda} u^{24 / 11}-\tilde{\Lambda} \omega^{24 / 11}\right\|\|v\|_{1} \\
& \quad \leqslant c k\left(2\left\|u^{12 / 11}-\omega^{12 / 11}\right\|+\left\|u^{0}-\omega^{0}\right\|\right)\|v\|_{1} \leqslant c k h^{r}\|v\|_{1} \\
& \quad \leqslant c \varepsilon^{-1} k h^{2 r}+\varepsilon k a(v, v) .
\end{aligned}
$$

From (2.8), (3.36), (3.37), (2.4) it follows for $\varepsilon>0$

$$
\begin{aligned}
& k\left|b\left(\tilde{\Lambda} u^{24 / 11}, \tilde{\Lambda} \omega^{24 / 11}-\tilde{\Lambda} U^{24 / 11}, v\right)\right| \\
& \quad \leqslant C k\left\|\tilde{\Lambda} u^{24 / 11}\right\|_{\infty}\left\|\tilde{\Lambda} \omega^{24 / 11}-\tilde{\Lambda} U^{24 / 11}\right\|\|v\|_{1} \\
& \quad \leqslant c k\left(2\left\|\zeta^{12 / 11}\right\|+\left\|\eta^{0}\right\|\right)\|v\|_{1} \leqslant c k\left(k^{3}+h^{r}\right)\|v\|_{1} \\
& \quad \leqslant c \varepsilon^{-1} k\left(k^{6}+h^{2 r}\right)+\varepsilon k a(v, v) .
\end{aligned}
$$

From (2.9), (3.46), for $\varepsilon^{\prime}>0$,

$$
\begin{aligned}
& k\left|b\left(u^{24 / 11}-\tilde{\Lambda} u^{24 / 11}, u^{24 / 11}, v\right)\right| \\
& \quad \leqslant C k\left\|u^{24 / 11}-\tilde{\Lambda} u^{24 / 11}\right\|\left\|u^{24 / 11}\right\|_{1, \infty}\|v\| \\
& \quad \leqslant c k^{3}\|v\| \leqslant c\left(\varepsilon^{\prime}\right)^{-1} k^{6}+\varepsilon^{\prime}\|v\|^{2} .
\end{aligned}
$$

From (2.7), (2.4), for $\varepsilon>0$,

$$
\begin{aligned}
& k\left|b\left(\tilde{\Lambda} u^{24 / 11}-\tilde{\Lambda} \omega^{24 / 11}, \tilde{\Lambda} u^{24 / 11}, v\right)\right| \\
& \quad \leqslant C k\left\|\tilde{\Lambda} u^{24 / 11}-\tilde{\Lambda} \omega^{24 / 11}\right\|\left\|\tilde{\Lambda} u^{24 / 11}\right\|_{1, \infty}\|v\|_{1} \\
& \quad \leqslant c k\left(2\left\|\eta^{12 / 11}\right\|+\left\|\eta^{0}\right\|\right)\|v\|_{1} \leqslant c k h^{r}\|v\|_{1} \\
& \quad \leqslant c \varepsilon^{-1} k h^{2 r}+\varepsilon k a(v, v) .
\end{aligned}
$$

From (2.7), (3.36), (3.37), (2.4), for $\varepsilon>0$,

$$
\begin{aligned}
& k\left|b\left(\tilde{\Lambda} \omega^{24 / 11}-\tilde{\Lambda} U^{24 / 11}, \tilde{\Lambda} u^{24 / 11}, v\right)\right| \\
& \quad \leqslant C k\left\|\tilde{\Lambda} \omega^{24 / 11}-\tilde{\Lambda} U^{24 / 11}\right\|\left\|\tilde{\Lambda} u^{24 / 11}\right\|_{1, \infty}\|v\|_{1} \\
& \quad \leqslant c k\left(2\left\|\zeta^{12 / 11}\right\|+\left\|\eta^{0}\right\|\right)\|v\|_{1} \leqslant c k\left(k^{3}+h^{r}\right)\|v\|_{1} \\
& \quad \leqslant c \varepsilon^{-1} k\left(k^{6}+h^{2 r}\right)+\varepsilon k a(v, v) .
\end{aligned}
$$

From (1.11), (2.4), for $\varepsilon>0$, since $r \geqslant 2$,

$$
\begin{aligned}
& k\left|b\left(\tilde{\Lambda} u^{24 / 11}-\tilde{\Lambda} \omega^{24 / 11}, \tilde{\Lambda} u^{24 / 11}-\tilde{\Lambda} \omega^{24 / 11}, v\right)\right| \\
& \quad \leqslant C_{b} k\left\|\tilde{\Lambda} u^{24 / 11}-\tilde{\Lambda} \omega^{24 / 11}\right\|_{1}^{2}\|v\|_{1} \\
& \quad \leqslant C k\left(\left\|\eta^{12 / 11}\right\|_{1}^{2}+\left\|\eta^{0}\right\|_{1}^{2}\right)\|v\|_{1} \leqslant c k h^{2 r-2}\|v\|_{1} \\
& \quad \leqslant c \varepsilon^{-1} k h^{4 r-4}+\varepsilon k a(v, v) \leqslant c \varepsilon^{-1} k h^{2 r}+\varepsilon k a(v, v)
\end{aligned}
$$


From (1.11), (3.36), (2.4), (1.5), (3.37) and the fact that $r \geqslant 2, h<1$, for $\varepsilon>0$,

$$
\begin{aligned}
k \mid b & \left(\tilde{\Lambda} u^{24 / 11}-\tilde{\Lambda} \omega^{24 / 11}, \tilde{\Lambda} \omega^{24 / 11}-\tilde{\Lambda} U^{24 / 11}, v\right) \mid \\
& \leqslant C_{b} k\left\|\tilde{\Lambda} u^{24 / 11}-\tilde{\Lambda} \omega^{24 / 11}\right\|_{1}\left\|\tilde{\Lambda} \omega^{24 / 11}-\tilde{\Lambda} U^{24 / 11}\right\|_{1}\|v\|_{1} \\
& \leqslant C_{b} k\left(2\left\|\eta^{12 / 11}\right\|_{1}+\left\|\eta^{0}\right\|_{1}\right)\left(2\left\|\zeta^{12 / 11}\right\|_{1}+\left\|\eta^{0}\right\|_{1}\right)\|v\|_{1} \\
& \leqslant c k h^{r-1}\left(h^{-1} k^{3}+h^{r-1}\right)\|v\|_{1} \leqslant c k\left(k^{3} h^{r-2}+h^{2 r-2}\right)\|v\|_{1} \\
& \leqslant c k\left(k^{3}+h^{2 r-2}\right)\|v\|_{1} \leqslant c \varepsilon^{-1} k\left(k^{6}+h^{4 r-4}\right)+\varepsilon k a(v, v) \\
& \leqslant c \varepsilon^{-1} k\left(k^{6}+h^{2 r}\right)+\varepsilon k a(v, v) .
\end{aligned}
$$

Exactly as in (3.54),

$$
\begin{aligned}
& k\left|b\left(\tilde{\Lambda} \omega^{24 / 11}-\tilde{\Lambda} U^{24 / 11}, \tilde{\Lambda} u^{24 / 11}-\tilde{\Lambda} \omega^{24 / 11}, v\right)\right| \\
& \leqslant C_{b} k\left\|\tilde{\Lambda} \omega^{24 / 11}-\tilde{\Lambda} U^{24 / 11}\right\|_{1}\left\|\tilde{\Lambda} u^{24 / 11}-\tilde{\Lambda} \omega^{24 / 11}\right\|_{1}\|v\|_{1} \\
& \leqslant c \varepsilon^{-1} k\left(k^{6}+h^{2 r}\right)+\varepsilon k a(v, v) .
\end{aligned}
$$

Finally, by (1.11), (3.36), (3.37), (1.5), (2.4), (3.13) and the facts that $h<1, r \geqslant 2$ we obtain for $\varepsilon>0$

$$
\begin{aligned}
& k\left|b\left(\tilde{\Lambda} \omega^{24 / 11}-\tilde{\Lambda} U^{24 / 11}, \tilde{\Lambda} \omega^{24 / 11}-\tilde{\Lambda} U^{24 / 11}, v\right)\right| \\
& \quad \leqslant C_{b} k\left\|\tilde{\Lambda} \omega^{24 / 11}-\tilde{\Lambda} U^{24 / 11}\right\|_{1}^{2}\|v\|_{1} \\
& \quad \leqslant C \varepsilon^{-1} k\left\|\tilde{\Lambda} \omega^{24 / 11}-\tilde{\Lambda} U^{24 / 11}\right\|_{1}^{4}+\varepsilon k a(v, v) \\
& \quad \leqslant C \varepsilon^{-1} k\left(\left\|\zeta^{12 / 11}\right\|_{1}^{4}+\left\|\eta^{0}\right\|_{1}^{4}\right)+\varepsilon k a(v, v) \\
& \quad \leqslant c \varepsilon^{-1} k\left[\left(k^{3} h^{-1}+h^{r-1}\right)^{4}+h^{4 r-4}\right]+\varepsilon k a(v, v) \\
& \leqslant c \varepsilon^{-1}\left(k^{13} h^{-4}+k h^{4 r-4}\right)+\varepsilon k a(v, v) \\
& \leqslant c \varepsilon^{-1}\left(k^{7} \alpha^{6}+k h^{2 r}\right)+\varepsilon k a(v, v) \\
& \leqslant c \varepsilon^{-1} k\left(k^{6}+h^{2 r}\right)+\varepsilon k a(v, v) .
\end{aligned}
$$

Hence, (3.45) and (3.47)-(3.56) give, for $\varepsilon, \varepsilon^{\prime}>0$,

$$
\begin{aligned}
k \mid b\left(u^{24 / 11}, u^{24 / 11}, v\right) & -b\left(2 U^{12 / 11}-u^{0}, 2 U^{12 / 11}-u^{0}, v\right) \mid \\
& \leqslant c\left(\left(\varepsilon^{\prime}\right)^{-1}+\varepsilon^{-1}\right)\left(k^{6}+h^{2 r}\right)+\varepsilon^{\prime}\|v\|^{2}+\varepsilon k a(v, v) .
\end{aligned}
$$

Now, (2.4) and Taylor's theorem with remainder in integral form give

$$
\begin{gathered}
\left\|\eta^{24 / 11}-\eta^{12 / 11}\right\| \leqslant c k h^{r} \\
\left\|u^{24 / 11}-u^{12 / 11}-\frac{6 k}{11}\left(u_{t}^{24 / 11}+u_{t}^{12 / 11}\right)\right\| \leqslant c k^{3} .
\end{gathered}
$$

Hence, putting $v=\hat{\zeta}_{3} \in \tilde{\mathbf{S}}$ in (3.39) and using the standard inequalities, we obtain, by (3.44), (3.57)-(3.59) for $\varepsilon, \varepsilon^{\prime}>0$,

$$
\begin{aligned}
\frac{1}{2}\left\|\hat{\zeta}_{3}\right\|^{2} & +\frac{6}{11} k \nu a\left(\hat{\zeta}_{3}, \hat{\zeta}_{3}\right) \\
& \leqslant \frac{1}{2}\left\|\zeta^{12 / 11}\right\|^{2}+c\left(\left(\varepsilon^{\prime}\right)^{-1}+\varepsilon^{-1}\right)\left(k^{6}+h^{2 r}\right)+\varepsilon^{\prime}\left\|\hat{\zeta}_{3}\right\|^{2}+\varepsilon k a\left(\hat{\zeta}_{3}, \hat{\zeta}_{3}\right),
\end{aligned}
$$

which in conjunction with (3.37) yields (3.38) for suitable $\varepsilon, \varepsilon^{\prime}$. 
We finally define $U^{24 / 11}$ by $(1.24)$ and obtain, if $\zeta^{24 / 11}=U^{24 / 11}-\omega^{24 / 11}$, by (3.38), (3.37)

$$
\begin{aligned}
\left\|\zeta^{24 / 11}\right\| & =\left\|2 \hat{U}_{3}-U^{24 / 11}-\omega^{24 / 11}\right\|=\left\|2 \hat{\zeta}_{3}+\omega^{12 / 11}-U^{12 / 11}\right\| \\
& \leqslant 2\left\|\hat{\zeta}_{3}\right\|+\left\|\zeta^{12 / 11}\right\| \leqslant c\left(k^{3}+h^{r}\right) .
\end{aligned}
$$

In particular, as a consequence of (3.37), (3.60) and (2.4) we see that our results imply (under the hypothesis (3.13)) that

$$
\begin{aligned}
& \left\|U^{12 / 11}-u^{12 / 11}\right\| \leqslant c\left(k^{3}+h^{r}\right), \\
& \left\|U^{24 / 11}-u^{24 / 11}\right\| \leqslant c\left(k^{3}+h^{r}\right) .
\end{aligned}
$$

The main result of this section may now be proved easily.

THEOREM 3.1. Let $U^{0}, U^{1}, U^{2}$ be defined by (1.18)-(1.26). Then $U^{j} \in \tilde{\mathbf{S}}$ for $j=0,1,2$. In addition, let (3.13) be satisfied for any positive constant $\alpha$. Then, there exists a constant $c=c(u, p, \alpha)$ such that

$$
\left\|U^{j}-u^{j}\right\| \leqslant c\left(k^{3}+h^{r}\right), \quad j=0,1,2 .
$$

In particular (taking into account (2.4)), (2.25) is satisfied for the initial values $U^{j}$, $j=0,1,2$.

Proof. The case $j=0$ has already been proved in (3.2). For $j=1$ (3.2), (3.61), (3.62) and Taylor's theorem with remainder in integral form yields

$$
\begin{aligned}
\left\|U^{1}-u^{1}\right\| \leqslant & \frac{1}{288}\left(13\left\|U^{0}-u^{0}\right\|+286\left\|U^{12 / 11}-u^{12 / 11}\right\|+11\left\|U^{24 / 11}-u^{24 / 11}\right\|\right) \\
& +\left\|\frac{1}{288}\left(13 u^{0}+286 u^{12 / 11}-11 u^{24 / 11}-288 u^{1}\right)\right\| \\
\leqslant & c\left(k^{3}+h^{r}\right)+c k^{3},
\end{aligned}
$$

thus proving (3.63) for $j=1$. The case $j=2$ is entirely analogous.

4. Remarks. 4.1. It is quite straightforward to show that, by choosing $u^{0}$ and $f$ sufficiently small in appropriate function spaces and/or $\nu$ sufficiently large, we can guarantee the validity of $(2.35)$ - more precisely $(2.43)$ - which we rewrite here as

$$
.\|u\|_{L^{\infty}\left(\mathbf{H}^{\prime}\right)} \leqslant \nu C_{a} / 660 C_{b} .
$$

The relevant estimates are easy to obtain, following, e.g., [12], [15], [18]. We state here the final results omitting the details of the proofs.

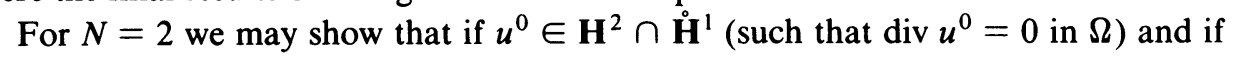
$f \in L^{\infty}\left(\mathbf{L}^{2}\right), f_{t} \in L^{2}\left(\mathbf{L}^{2}\right)$, then

$$
\begin{aligned}
\|u\|_{L^{\infty}\left(\mathbf{H}^{1}\right)} \leqslant & \exp \left(\frac{\left\|u^{0}\right\|^{2}}{\nu^{2} C_{a}^{2}}+\frac{\|f\|_{L^{2}\left(\mathbf{L}^{2}\right)}^{2}}{\nu^{3} C_{a}^{3}}\right) \\
& \times\left(\frac{\sqrt{2}}{C_{a}}\left\|u^{0}\right\|_{2}+\frac{C_{*}}{\nu C_{a}}\left\|u^{0}\right\|_{1}\left\|u^{0}\right\|_{2}\right. \\
& \left.\quad+\frac{1}{\nu C_{a}}\|f(0)\|+\frac{1}{\left(\nu C_{a}\right)^{3 / 2}}\left\|f_{t}\right\|_{L^{2}\left(\mathbf{L}^{2}\right)}\right) \\
& +\frac{1}{\nu C_{a}}\|f\|_{L^{\infty}\left(\mathbf{L}^{2}\right)},
\end{aligned}
$$


where $C_{*}$ is a constant depending on $\Omega$ only. It is clear then that (4.1) may be satisfied for $\nu$ sufficiently large and/or $u^{0}, f$ small in an appropriate sense.

For $N=3$, following, e.g., the analysis of [18, p. 303 et seq.] one may show in particular the following type of result-cf. also Remark 2.1 -especially suitable to guarantee the validity of (2.43). Under the previous hypotheses on $u^{0}, f$, suppose that for some $\varepsilon>0$,

$$
\begin{aligned}
\frac{\|f\|_{L^{\infty}\left(\mathbf{L}^{2}\right)}^{2}}{\nu C_{a}} & +\left[1+\left(\sqrt{3} \nu\left\|u^{0}\right\|_{2}+C^{*}\left\|u^{0}\right\|_{2}^{2}+\|f(0)\|^{2}\right)\right] \\
& \times\left(\left\|u^{0}\right\|^{2}+\frac{T\|f\|_{L^{\infty}\left(\mathbf{L}^{2}\right)}^{2}}{\nu C_{a}}\right)^{1 / 2} \exp \left(\left\|f_{t}\right\|_{L^{1}\left(\mathbf{L}^{2}\right)}\right)<\frac{\nu^{3} C_{a}^{2}}{\varepsilon^{2} C_{b}^{2}},
\end{aligned}
$$

where $C^{*}=C^{*}(\Omega)$. (Note that for each $\varepsilon>0$ and fixed $T$ (4.3) can be guaranteed again for $\nu$ sufficiently large and/or $u^{0}, f$ appropriately small.) It follows then that

$$
\|u\|_{L^{\infty}\left(\mathbf{H}^{1}\right)} \leqslant \frac{\nu C_{a}}{\varepsilon C_{b}}
$$

which is (4.1) if $\varepsilon=660$.

4.2. Instead of the third order accurate backward differentiation method used here one may also use the two-step, second order accurate backward differentiation method, which, in the case $y^{\prime}=F(y)$, becomes

$$
y^{n+2}-\frac{4}{3} y^{n+1}+\frac{1}{3} y^{n}=\frac{2}{3} k F^{n+2}, \quad 0 \leqslant n \leqslant J-2, y^{0}, y^{1} \text { given. }
$$

Introducing now the second order "lag" operator $\bar{\Lambda}$ by

$$
\bar{\Lambda} y^{n}=2 y^{n-1}-y^{n-2}, \quad 2 \leqslant n \leqslant J
$$

we may formulate a second order backward differentiation-Galerkin method as follows: seek $\left\{\left[U^{n}, P^{n}\right]\right\}, 2 \leqslant n \leqslant J$ in $\mathbf{H}_{h, \hat{h}}$ satisfying

$$
\begin{aligned}
\left(U^{n+2}-\right. & \left.\frac{4}{3} U^{n+1}+\frac{1}{3} U^{n}, v\right)+\frac{2}{3} k B\left(\left[U^{n+2}, P^{n+2}\right],[v, q]\right) \\
+\frac{2}{3} k b\left(\bar{\Lambda} U^{n+2}, \bar{\Lambda} U^{n+2}, v\right)=\frac{2}{3} k\left(f^{n+2}, v\right), & 0 \leqslant n \leqslant J-2, \\
& \forall[v, q] \in \mathbf{H}_{h, \hat{h}} .
\end{aligned}
$$

The initial conditions $U^{0}, U^{1}$ are chosen as follows. First we put $U^{0}=u^{0}$. (It is not necessary that $U^{0}$ be in $\mathbf{S}_{h}^{r}$.) We then define two intermediate values $\left[U^{2 / 3}, P^{2 / 3}\right]$, $\left[U^{4 / 3}, P^{4 / 3}\right]$ in $\mathbf{H}_{h, \hat{h}}$, with two consecutive applications of the backward Euler method, by

$$
\begin{aligned}
\left(U^{2 / 3}-u^{0}, v\right)+\frac{2}{3} k B\left(\left[U^{2 / 3}, P^{2 / 3}\right],[v, q]\right) & +\frac{2}{3} k b\left(u^{0}, u^{0}, v\right) \\
& =\frac{2}{3} k\left(f^{2 / 3}, v\right), \quad \forall[v, q] \in \mathbf{H}_{h, \hat{h}}, \\
\left(U^{4 / 3}-U^{2 / 3}, v\right)+\frac{2}{3} k B\left(\left[U^{4 / 3}, P^{4 / 3}\right],[v, q]\right)+\frac{2}{3} k b\left(u^{0}, u^{0}, v\right) & \\
= & \frac{2}{3} k\left(f^{4 / 3}, v\right), \quad \forall[v, q] \in \mathbf{H}_{h, \hat{h}},
\end{aligned}
$$


and finally define

$$
U^{1}=\frac{1}{2}\left(U^{2 / 3}+U^{4 / 3}\right) .
$$

It can be seen, using the methods of Section 3, albeit in a much more straightforward manner, that

$$
\left\|U^{1}-u^{1}\right\| \leqslant c\left(k^{2}+h^{r}\right),
$$

unconditionally. Using now the methods of Section 2 , appropriately simplified, we can show that if $U^{0}, U^{1}$ are chosen as above, if $k h^{-4 / 5} \leqslant \alpha$ for any $\alpha>0$, and if a condition of the form (2.35) holds, then, for $k$ and $h$ sufficiently small (specifically if $\left.c\left(k^{2}+h^{r-1}\right) \leqslant \nu, c T\left(\alpha^{4} h^{6 / 5}+h^{2 r-2}\right) \leqslant \nu\right)$, it follows that

$$
\left\|U^{n}-u^{n}\right\| \leqslant c(1+T)^{1 / 2}\left(k^{2}+h^{r}\right), \quad 2 \leqslant n \leqslant J .
$$

Again, if (2.35) is not required to hold we may obtain again the analog of (2.65), i.e., that

$$
\left\|U^{n}-u^{n}\right\| \leqslant c e^{c T}\left(k^{2}+h^{r}\right), \quad 2 \leqslant n \leqslant J,
$$

for $k, h$ appropriately small. For the pressure we obtain now that $\left|P^{n}-p^{n}\right|_{L^{2} / R^{1}}=$ $O\left(k+k^{-1} h^{r}+h^{r-1}\right), 2 \leqslant n \leqslant J$.

4.3. For the proof of Proposition 2.1 we need a preliminary result.

LeMma 4.1. Let $p \in H^{1} / R^{1}$. Then, there exists a unique $b \in \mathbf{H}^{2} \cap \stackrel{\mathrm{H}}{1}^{1}$ satisfying

$$
\begin{cases}\nu \Delta b=\operatorname{grad} p & \text { in } \Omega, \\ b=0 & \text { on } \partial \Omega .\end{cases}
$$

Moreover, for positive constants $C_{1}^{\prime}, C_{2}^{\prime}, C_{3}^{\prime}$, where $C_{1}^{\prime}=C(\Omega) / \nu, C_{2}^{\prime}=C(N) \nu$, $C_{3}^{\prime}=C(\Omega) \nu$, we have

$$
\|b\|_{2} \leqslant C_{1}^{\prime}\left[\sum_{i=1}^{N} \int_{\Omega}\left(\frac{\partial p}{\partial x_{i}}\right)^{2} d x\right]^{1 / 2}
$$

and

$$
C_{2}^{\prime} a(b, b)^{1 / 2} \leqslant|p|_{L^{2} / R^{1}} \leqslant C_{3}^{\prime} a(b, b)^{1 / 2}
$$

Proof. Standard elliptic existence and regularity theory shows that the solution $b$ of (4.6) exists uniquely and satisfies (4.7). Moreover, for any real constant $\lambda$

$$
\begin{aligned}
\nu a(b, v) & =-(b, \nu \Delta b)=(-b, \operatorname{grad} p)=-(b, \operatorname{grad}(p+\lambda)) \\
& =\langle\operatorname{div} b, p+\lambda\rangle \leqslant|p+\lambda||\operatorname{div} b| \leqslant C(N)|p|_{L^{2} / R^{1}} a(b, b)^{1 / 2},
\end{aligned}
$$

from which the first inequality of (4.8) follows.

Now note that if $\mu(\Omega)=\int_{\Omega} d x$, then

$$
\begin{aligned}
|p|_{L^{2} / R^{1}} & =\inf _{\lambda \in R^{1}}|p+\lambda| \leqslant\left|p-(\mu(\Omega))^{-1} \int_{\Omega} p d x\right| \\
& =\sup _{0 \neq g \in L^{2}}\left\langle p-(\mu(\Omega))^{-1} \int_{\Omega} p d x, g\right\rangle /|g| .
\end{aligned}
$$


Consider the boundary value problem

$$
\begin{cases}-\nu \Delta \psi+\operatorname{grad} \rho=0 & \text { in } \Omega, \\ \psi=0 & \text { on } \partial \Omega \\ \operatorname{div} \psi=D & \text { in } \Omega .\end{cases}
$$

It is known, cf. [18, Proposition 2.3, p. 35], that if $D \in L^{2}$ is such that $\int_{\Omega} D d x=0$, then, (4.10) has a unique solution $[\psi, \rho] \in \stackrel{\mathrm{H}}{ }^{1} \times L^{2} / R^{1}$. Moreover it can be seen that, for some constant $C=C(\Omega)$,

$$
\nu\|\psi\|_{1}+|\rho|_{L^{2} / R^{1}} \leqslant C \nu|D| \text {. }
$$

Let now $g \in L^{2}$ and $[\psi, \rho]$ satisfy (4.10) with $D=g-(\mu(\Omega))^{-1} \int_{\Omega} g d x$. There follows that

$$
\begin{gathered}
\left\langle p-(\mu(\Omega))^{-1} \int_{\Omega} p d x, g\right\rangle=\langle p, \operatorname{div} \psi\rangle=-(\psi, \operatorname{grad} p) \\
=-(\psi, \nu \Delta b)=\nu a(\psi, b) \leqslant \nu a(\psi, \psi)^{1 / 2} a(b, b)^{1 / 2} \\
\leqslant \nu\|\psi\|_{1} a(b, b)^{1 / 2} \leqslant C \nu|g| a(b, b)^{1 / 2}
\end{gathered}
$$

for some constant $C=C(\Omega)$; here (4.11) was used in the last inequality. (4.9) and (4.12) yield now the second inequality of (4.8).

Proof of Proposition 2.1. (2.1) follows in a straightforward manner from the Cauchy-Schwartz inequality. To prove (2.2), let $[u, p] \in \mathbf{H}_{h, \hat{h}}$. Let then $b$ satisfy (4.6) and $\chi \in \mathbf{S}_{h}^{r}$ be such that

$$
\|b-\chi\|_{1} \leqslant C h\|b\|_{2} .
$$

For any $q \in P_{\hat{h}}^{r} / R^{1}$ we have, as a consequence of (4.6), that

$$
\begin{aligned}
B([u, p],[u-\chi, q])= & \nu a(u, u)-\nu a(u, \chi-b)+(u, \operatorname{grad} p) \\
& +(u, \operatorname{grad}(p+q))-(\chi-b, \operatorname{grad} p)+\nu a(b, b) .
\end{aligned}
$$

Putting $q=-2 p$ in the above and using (1.3) we obtain, for some constant $C=C(N)$, that

(4.14) $B([u, p],[u-\chi,-2 p])$

$$
\begin{aligned}
& =\nu a(u, u)-\nu a(u, \chi-b)+\langle\operatorname{div}(\chi-b), p\rangle+\nu a(b, b) \\
& \geqslant \nu C_{a}\|u\|_{1}^{2}-\nu\|u\|_{1}\|b-\chi\|_{1}-C\|b-\chi\|_{1}|p|_{L^{2} / R^{1}}+\nu a(b, b) .
\end{aligned}
$$

Now, from (4.13), (4.7), (1.7) there follows

$$
\|b-\chi\|_{1} \leqslant \frac{C}{\nu}\left(\frac{h}{\hat{h}}\right)|p|_{L^{2} / R^{1}}
$$

Also, from (4.8) we obtain

$$
\nu a(b, b) \geqslant \frac{C}{\nu}|p|_{L^{2} / R^{1}}^{2}
$$

In view of the above (4.14) gives, for some positive constants $\tilde{C}_{i}, i=1,2,3$ depending on $\Omega$ only, and for any $\varepsilon>0$, that

$$
\begin{aligned}
B([u, p],[u-\chi,-2 p]) \\
\geqslant\left(\nu C_{a}-\varepsilon\right)\|u\|_{1}^{2}+\left[\frac{\tilde{C}_{1}}{\nu}-\frac{\tilde{C}_{2}}{\nu}\left(\frac{h}{\hat{h}}\right)-\frac{\tilde{C}_{3}}{\varepsilon}\left(\frac{h}{\hat{h}}\right)^{2}\right]|p|_{L^{2} / R^{1}}^{2}
\end{aligned}
$$


Pick now, e.g., $\varepsilon=\nu C_{a} / 2$. Then, given any positive constant $\lambda<\tilde{C}_{1}$, it is possible to choose a constant $\beta$ so that

$$
\tilde{C}_{1}-\tilde{C}_{2}\left(\frac{h}{\hat{h}}\right)-\frac{2 \tilde{C}_{3}}{C_{a}}\left(\frac{h}{\hat{h}}\right)^{2}>\lambda>0,
$$

for $0<h / \hat{h} \leqslant \beta$. For such $h / \hat{h}$ we conclude therefore that

$$
B([u, p],[u-\chi,-2 p]) \geqslant C(\beta, \nu, \Omega)_{\|[}[u, p]_{\|\|^{2}} .
$$

Now note, using (1.3), (4.8), (4.16) and the fact that $h / \hat{h} \leqslant \beta$, that

$$
\|u-\chi\|_{1} \leqslant\|u\|_{1}+\|b\|_{1}+\|b-\chi\|_{1} \leqslant\|u\|_{1}+C(\beta, \nu, \Omega)|p|_{L^{2} / R^{1}} .
$$

We conclude then that, for some constant $C=C(\beta, \nu, \Omega)$,

$$
\|[u-\chi,-2 p]\| \leqslant C\|[u, p]\| .
$$

Hence (4.16) and (4.17) yield

$$
\begin{aligned}
\sup _{\substack{[v, q] \in \mathbf{H}_{h, \hat{h}} \\
\|[v, q]\| \leqslant 1}}|B([u, p],[v, q])| & \geqslant B([u, p],[u-\chi,-2 p]) /\|[u-\chi,-2 p]\| \\
& \geqslant C_{2}(\beta, \nu, \Omega)_{\|}[u, p] \|,
\end{aligned}
$$

which is precisely (2.2).

Proof of Proposition 2.2. We suppress the $t$-dependence to simplify notation. The existence and uniqueness of $[\omega, \pi]$ satisfying (2.3), and the estimates (2.4) for $\mu=1$, $j=0$ and (2.5) follow in a straightforward manner from the properties of the bilinear form $B$ proved in Proposition 2.1 and the approximation properties (1.4) and (1.6). For a proof in a more abstract setting (motivated by analogous results of [1]), cf. Theorem 3.1 of an article by the first author in Math. Comp., v. 29, 1975, p. 999.

To prove (2.4) for $\mu=0, j=0$, one may use the classic Aubin-Nitsche duality argument as follows. Put $e=u-\omega, \varepsilon=p-\pi$, and let $\phi \in\left(C^{\infty}(\Omega)\right)^{N}$. Consider the boundary value problem

$$
\begin{cases}-\nu \Delta \psi+\operatorname{grad} \rho=\phi & \text { in } \Omega, \\ \psi=0 & \text { on } \partial \Omega, \\ \operatorname{div} \psi=0 & \text { in } \Omega,\end{cases}
$$

which, cf. [18, Proposition 2.3, p. 35], for each integer $m \geqslant-1$, has a unique solution $[\psi, \rho] \in \stackrel{\mathbf{H}}{ }^{1} \cap \mathbf{H}^{m+2} \times H^{m+1} / R^{1}$ that satisfies the estimate

$$
\|\psi\|_{m+2}+|\rho|_{H^{m+1} / R^{\prime}} \leqslant C_{m}\|\phi\|_{m} .
$$

Using (4.18), the symmetry of $B$ and (2.3) yields

$$
\begin{aligned}
(e, \phi) & =B([\psi, \rho],[e, \varepsilon])=B([e, \varepsilon],[\psi, \rho]) \\
& =B([e, \varepsilon],[\psi, \rho]-[v, q]), \quad \forall[v, q] \in \mathbf{H}_{h, \hat{h}}
\end{aligned}
$$

Hence, in view of (2.1), the first part of this proof and (4.19) with $m=0$ yield

$$
\begin{aligned}
&|(e, \phi)| \leqslant C_{1}\left\|[e, \varepsilon]_{\|} \inf _{[v, q] \in \mathbf{H}_{h, \hat{h}}}\right\|[\psi, \rho]-[v, q]_{\|} \\
& \leqslant C h\left(\|\psi\|_{2}+|\rho|_{H^{1} / R^{1}}\right)\left\|[e, \varepsilon]_{\|} \leqslant C h\right\| \phi\|\|[e, \varepsilon]_{\|} .
\end{aligned}
$$

It follows that

$$
\|e\| \leqslant C h \|[e, \varepsilon]_{\|},
$$


from which we obtain (2.4) for $\mu=0, j=0$. The case $j=1$ follows easily by noting that $\left[\omega_{t}, \pi_{t}\right]$ is the $B$-projection of $\left[u_{t}, p_{t}\right]$.

4.4. The restriction of having the subspace $\mathbf{S}_{h}^{r}$ satisfy the zero boundary conditions may be alleviated, as done in [2], by using other nonstandard Galerkin methods, e.g., similar techniques to the ones used by Nitsche [16], [17] for the Dirichlet problem or by using subspaces satisfying "nearly zero" boundary conditions [17]. Following [2], for $r \geqslant 2$ we may let, e.g., $\mathbf{S}_{h}^{r}$ be a one-parameter family of subspaces of $\mathbf{H}^{1}(\Omega)$ consisting of $N$-tuples of polynomials of degree at most $r-1$ satisfying certain approximation and inverse properties, cf. [2, Section 2] for details, and $P_{\hat{h}}^{r}$ be the pressure space as in Section 1. Letting again $\mathbf{H}_{h, \hat{h}}=\mathbf{S}_{h}^{r} \times P_{\hat{h}}^{r} / R^{1}$ we now use, for the space approximation, instead of $B(\cdot, \cdot)$, the bilinear form $B^{\gamma}(\cdot, \cdot)$, defined on $\mathbf{H}_{h, \hat{h}} \times \mathbf{H}_{h, \hat{h}}$ as

$$
\begin{aligned}
B^{\gamma}([u, p],[v, q])= & B([u, p],[v, q]) \\
& -\int_{\partial \Omega}\left(\nu u \frac{\partial v}{\partial n}+\nu v \frac{\partial u}{\partial n}-\gamma h^{-1} u v\right) d \sigma,
\end{aligned}
$$

where $\partial u / \partial n$ is the outward normal derivative of $u$ on $\partial \Omega$ and $\gamma>0$ is a suitably chosen parameter. We refer the reader to [2] for the analogs of Propositions 2.1 and 2.2. The time-stepping method and its analysis proceed then in an entirely analogous manner to what was done here, yielding, e.g., the optimal $\mathbf{L}^{2}$-error estimate $\| U^{n}-$ $u^{n} \|=O\left(k^{3}+h^{r}\right), 0 \leqslant n \leqslant J$, under similar hypotheses on $k, h$ and $u$. The method may be further extended to the case of nonhomogeneous boundary conditions, e.g., with $u(x, t)$ prescribed on $\partial \Omega \times[0, T]$ but not necessarily equal to zero; cf. [2].

Laboratorio de Computacao Cientificas- $\mathrm{CNP}_{\mathrm{q}}$

Av. Wenceslau Braz. 71

22290 Rio de Janeiro-R. J., Brazil

Department of Mathematics

University of Tennessee

Knoxville, Tennessee 37996

Department of Mathematics

University of Tennessee

Knoxville, Tennessee 37996

1. I. BABUSKA, "Survey lectures on the mathematical foundations of the finite element method" in The Mathematical Foundations of the Finite Element Method with Applications to Partial Differential Equations (A. K. Aziz, Ed.), Academic Press, New York and London, 1972.

2. G. A. BAKER, "Galerkin approximations to the Navier-Stokes equations." (To appear.)

3. J. H. BRAMBLE, "Multistep methods for quasilinear parabolic equations," in Computational Methods in Nonlinear Mechanics (J. T. Oden, Ed.), Elsevier-North-Holland, New York, 1980.

4. J. H. Bramble \& P. H. Sammon, "Efficient higher order single step methods for parabolic problems: Part I," Math. Comp., v. 35, 1980, pp. 655-677.

5. M. Crouzeix \& P.-A. Raviart, "Conforming and nonconforming finite element methods for solving the stationary Stokes equations," RAIRO Anal. Numér., v. 7-R3, 1973, pp. 33-76.

6. J. Douglas, JR., T. Dupont \& R. Ewing, "Incomplete iteration for time-stepping a Galerkin method for a quasilinear parabolic problem," SIAM J. Numer. Anal., v. 16, 1979, pp. 503-522.

7. T. Dupont, G. Fairweather \& J. P. Johnson, "Three-level Galerkin methods for parabolic equations," SIAM J. Numer. Anal., v. 11, 1974, pp. 392-410.

8. R. S. FALK, "An analysis of the finite element method using Lagrange multipliers for the stationary Stokes equations," Math. Comp., v. 30, 1976, pp. 241-249. 
9. V. Girault \& P.-A. Raviart, Finite Element Approximation of the Navier-Stokes Equations, Lecture Notes in Math., vol. 749, Springer-Verlag, Berlin and New York, 1979.

10. P. JAMET \& P.-A. RAviart, “Numerical solution of the stationary Navier-Stokes equations by finite element methods," in International Symposium on Computing Methods in Applied Sciences and Engineering IRIA 1973, (R. Glowinski and J. L. Lions, Eds.), Lecture Notes in Comput. Sci., vol. 10, Springer-Verlag, Berlin and New York, 1974, pp. 193-223.

11. W. Jureidini, A Mathematical Study of Two-Dimensional Incompressible Viscous Flows, Ph.D. Thesis, Harvard University, Cambridge, Mass., 1980.

12. O. A. Ladyzhenskaya, The Mathematical Theory of Viscous, Incompressible Flow, 2nd ed., Gordon and Breach, New York, 1969.

13. J. D. LAmberT, Computational Methods in Ordinary Differential Equations, Wiley, New York, 1973.

14. M.-N. LE Roux, "Méthodes multipas pour des équations paraboliques non linéaires," Numer. Math., v. 35, 1980, pp. 143-162.

15. J. L. Lions, Quelques Méthodes de Résolution des Problèmes aux Limites Non Linéaires, Dunod, Gauthier-Villars, Paris, 1969.

16. J. Nitsche, “Über ein Variationsprinzip zur Lösung von Dirichlet-Problemen bei Verwendung von Teilräumen, die keinen Randbedingungen unterworfen sind," Abh. Math. Sem. Univ. Hamburg, v. 36, 1971, pp. 9-15.

17. J. NiTSCHE, “On Dirichlet problems using subspaces with nearly zero boundary conditions," in The Mathematical Foundations of the Finite Element Method with Applications to Partial Differential Equations, (A. K. Aziz, Ed.), Academic Press, New York, 1972, pp. 603-627.

18. R. Temam, Navier-Stokes Equations. Theory and Numerical Analysis, rev. ed., North-Holland, Amsterdam, 1979.

19. M. Zlámal, "Finite element methods for nonlinear parabolic equations," RAIRO Anal. Numér., v. 11, 1977, pp. 93-107. 